\title{
$\beta$-decay properties of ${ }^{72} \mathrm{Ni}$ and ${ }^{72} \mathrm{Cu}$
}

\author{
J.-C. Thomas, ${ }^{*}$ H. De Witte, M. Gorska, ${ }^{\dagger}$ M. Huyse, K. Kruglov, Y. Kudryavtsev, D. Pauwels, N. V. S. V. Prasad, \\ K. Van de Vel, ${ }^{\ddagger}$ P. Van Duppen, and J. Van Roosbroeck \\ Instituut voor Kern- en Stralingsfysica, University of Leuven, Celestijnenlaan 200D, B-3001 Leuven, Belgium \\ S. Franchoo, ${ }^{\S}$ J. Cederkall, H. O. U. Fynbo," U. Georg, O. Jonsson, and U. Köster \\ ISOLDE, CERN, CH-1211 Genève 23, Switzerland \\ L. Weissman and W. F. Mueller \\ National Superconducting Cyclotron Laboratory, Michigan State University, 164 S. Shaw Lane, 48824-1312 Michigan, USA \\ V. N. Fedosseev** and V. I. Mishin \\ Institute of Spectroscopy, Russian Academy of Sciences, RU-142092 Troitsk, Russia \\ D. Fedorov \\ St. Petersburg Nuclear Physics Institute, RU-188350 Gatchina, Russia
}

A. De Maesschalck and N. A. Smirnova

Vakgroep Subatomaire en Stralingsfysica, Universiteit Gent, Proeftuinstraat 86, B-9000 Gent, Belgium

(Received 1 September 2005; revised manuscript received 6 August 2006; published 21 November 2006)

\begin{abstract}
The $\beta$-decay properties of ${ }_{28}^{72} \mathrm{Ni}_{44}$ and ${ }_{29}^{72} \mathrm{Cu}_{43}$ have been studied at the LISOL facility of Louvain-La-Neuve and at the CERN-ISOLDE facility, respectively. These neutron-rich nuclei have been produced in the proton-induced fission of ${ }^{238} \mathrm{U}$. Their decay schemes are presented and the lifetime $T_{1 / 2}=6.63(3) \mathrm{s}$ of ${ }^{72} \mathrm{Cu}$ was measured. No $\beta$-decaying isomeric state was found in ${ }^{72} \mathrm{Cu}$, in line with a suggested spin (2) for its ground state. Spin and parity assignments of the observed excited states in the odd-odd nucleus ${ }^{72} \mathrm{Cu}$ are proposed and discussed in terms of coupling between the valence proton and neutrons. Comparison is made with a schematic shell-model picture of ${ }^{72} \mathrm{Cu}$ and with large-scale shell-model calculations performed in the $\left(2 p_{3 / 2} 1 f_{5 / 2} 2 p_{1 / 2} 1 g_{9 / 2}\right)$ shell space outside the doubly magic ${ }_{28}^{56} \mathrm{Ni}_{28}$ core.
\end{abstract}

DOI: 10.1103/PhysRevC.74.054309 PACS number(s): 21.10.-k, 23.40.-s, 25.85.Ge, 27.50.+e

\section{INTRODUCTION}

\section{A. Motivation}

The nuclear structure and the decay properties of neutronrich nuclei in the vicinity of the magic nucleus ${ }_{28}^{68} \mathrm{Ni}_{40}$ have been extensively investigated in the past few years (see [1] and references therein). The aim of these studies is to evaluate the strength of the neutron subshell closure at $N=40$ and, generally speaking, to test the reliability of nuclear shell models in this far-off stability region. Depending on the robustness of the neutron subshell closure at $N=40$,

\footnotetext{
*Electronic address: thomasjc@ganil.fr; present address: GANIL, B.P. 55027, F-14076 Caen Cedex 5, France.

${ }^{\dagger}$ Present address: GSI, Planckstrasse 1, D-64291 Darmstadt, Germany.

${ }^{\ddagger}$ Present address: VITO, IMS, Mol, Belgium.

$\S$ Present address: IPN Orsay, 15 rue G. Clémenceau, F-91406 Orsay Cedex, France.

"Also at Department of Physics and Astronomy, University of Århus, Dk-8000 Århus C, Denmark.

"Present address: Institut Laue-Langevin, 6 rue J. Horowitz, BP 156, F-38042 Grenoble, France.

**Also at CERN, 1211 Genève 23, Switzerland.
}

collective driving nucleon-nucleon forces are indeed expected to balance shell effects in nuclei having $Z \approx 28$ protons and $N=40$ to 50 neutrons.

In addition, the decay properties and the nuclear structure of the neutron-rich nuclei lying between ${ }^{68} \mathrm{Ni}$ and the doubly magic ${ }_{28}^{78} \mathrm{Ni}_{50}$ can be relevant for the modelization of astrophysical processes $[2,3]$.

Copper isotopes with $A>69$ are good candidates to study the strength and the evolution of nuclear shell effects since their excited states may be partly described in terms of particle-particle and particle-hole couplings between the valence proton and neutrons [4]. The systematic study of the nuclear structure of the odd ${ }^{69,71,73} \mathrm{Cu}$ isotopes has already stressed the prominent role of the monopole part of the residual proton-neutron interaction [5]. In a recent work, the low-energy spectrum of ${ }^{70} \mathrm{Cu}$ has as well been successfully described by means of large-scale shell-model calculations in terms of coupling between the valence proton and the valence neutron [6,7]. Extended nuclear structure studies in this mass region should therefore lead to a better understanding of the residual proton-neutron interaction.

In the following, we will discuss new results obtained in $\beta$-decay studies of ${ }^{72} \mathrm{Ni}$ and ${ }^{72} \mathrm{Cu}$ at the LISOL facility of the Centre de Recherche du Cyclotron at Louvain-La-Neuve and 
at the ISOLDE facility of CERN, respectively. Data related to the $\beta$ decay of ${ }^{72} \mathrm{Cu}$ comprise part of a systematic study of the decay properties of very neutron rich copper isotopes. The decay properties of ${ }^{74,76,78} \mathrm{Cu}$ are discussed in [8] and the one of ${ }^{71,73,75,77} \mathrm{Cu}$ will be presented in a forthcoming article [9].

\section{B. Previous work}

The $\beta$ decay of ${ }^{72} \mathrm{Cu}$ was first investigated 20 years ago by Runte et al. [10]. In this work, ${ }^{72} \mathrm{Cu}$ was produced by means of multinucleon transfer reactions. A half-life of 6.6(1) s and a partial decay scheme toward excited states in the daughter nucleus ${ }^{72} \mathrm{Zn}$ were reported. Additional information on the nuclear structure of ${ }^{72} \mathrm{Zn}$ results from an earlier ${ }^{70} \mathrm{Zn}(t, p)^{72} \mathrm{Zn}$ transfer reaction work by Hudson and Glover [11] and from multinucleon transfer reactions performed recently by Wilson et al. [12].

${ }^{72} \mathrm{Ni}$ was first identified by Armbruster et al. in the thermalneutron-induced fission of ${ }^{235} \mathrm{U}$ [13]. A recent $\beta$-decay study performed at the LISOL facility of Louvain-La-Neuve by Franchoo et al. [14] led to a lifetime value of 1.57(5) s, in agreement with the first measurement of 2.20(41) s reported in [15]. A fragmentation study by Grzywacz et al. [16] led to the identification of a microsecond isomeric state at $270 \mathrm{keV}$ in ${ }^{72} \mathrm{Cu}$. In that work, three $\gamma$ transitions at 51,82 , and $138 \mathrm{keV}$ were associated with the decay of the isomeric state toward the ground state of ${ }^{72} \mathrm{Cu}$. These three $\gamma$ transitions were also identified in a fragmentation reaction experiment performed at the GANIL Facility $[17,18]$. The spin and parity of the ground state and of the three involved excited states were derived from half-life measurements and multipolarity considerations.

\section{EXPERIMENTAL CONDITIONS}

\section{A. Production methods and detection setups}

In the present work, ${ }^{72} \mathrm{Ni}$ and ${ }^{72} \mathrm{Cu}$ have been studied by means of the ISOL technique in a four-step process consisting of (i) the fission of ${ }^{238} \mathrm{U}$ induced by a proton beam of several $\mu \mathrm{A}$, (ii) the selective laser ionization of $\mathrm{Ni}$ or $\mathrm{Cu}$ atoms inside a laser ionization source, (iii) the mass separation of the selected ions, and (iv) the implantation of the separated ions on a movable tape surrounded by $\beta$ and $\gamma$ detection devices. ${ }^{72} \mathrm{Cu}$ was produced at the ISOLDE facility whereas ${ }^{72} \mathrm{Ni}$ was studied at the LISOL facility. In the first case, a $1-\mathrm{GeV}$ proton beam of $2-\mu \mathrm{A}$ average beam intensity was impinged on a thick $\mathrm{UC}_{x} /$ graphite target of $50 \mathrm{~g} / \mathrm{cm}^{2}$; in the latter case, a $30-\mathrm{MeV}$ proton beam of $6.4 \mu \mathrm{A}$ was used with two thin ${ }^{238} \mathrm{U}$ targets of $10 \mathrm{mg} / \mathrm{cm}^{2}$ tilted at $20^{\circ}$ with respect to the beam and placed in a 500-mbar Ar gas cell.

For the decay study of ${ }^{72} \mathrm{Cu}$ at ISOLDE, two separate detection setups were used. In the first one, three thin plastic $\Delta E$ detectors and two $\mathrm{HPGe}$ detectors of $64 \%$ and $90 \%$ relative efficiency were used in close geometry. In the second setup devoted to lifetime measurements, the activity was deposited on a movable tape passing through a $4 \pi \beta$ detector. The $\beta-\gamma$ decay of ${ }^{72} \mathrm{Ni}$ was observed at LISOL by means of four plastic
TABLE I. Experimental conditions for the decay study of ${ }^{72} \mathrm{Ni}$ and ${ }^{72} \mathrm{Cu}$. For each nucleus, the time structure (implantation and decay periods) of the cycles is given as well as the number of cycles after which the tape was moved. The total measurement time with and without laser ionization is shown in the next column. The last column gives the average production yields obtained for the two selectively ionized isotopes.

\begin{tabular}{lcrccc}
\hline \hline Nucleus & $\begin{array}{c}\text { Cycle } \\
\text { (impl./dec.) }\end{array}$ & Tape & Laser on & $\begin{array}{c}\text { Laser } \\
\text { off }\end{array}$ & $\begin{array}{c}\text { Yield } \\
\text { (ions } / \mu \mathrm{C})\end{array}$ \\
\hline${ }^{72} \mathrm{Ni}$ & $0.6 \mathrm{~s} / 0 \mathrm{~s}$ & 150 & $13 \mathrm{~h} 14 \mathrm{~min}$ & $50 \mathrm{~min}$ & $\sim 10$ \\
${ }^{72} \mathrm{Cu}$ & $0.5 \mathrm{~s} / 7 \mathrm{~s}$ & 1 & $20 \mathrm{~min}$ & - & $1.3 \times 10^{7}$ \\
\hline \hline
\end{tabular}

$\Delta E$ detectors, three of them being associated with $\mathrm{HPGe}$ detectors of $70 \%, 75 \%$, and $90 \%$ relative efficiency.

Table I gives a summary of the experimental conditions for the decay study of ${ }^{72} \mathrm{Ni}$ and ${ }^{72} \mathrm{Cu}$ : Each run consisted in the repetition of cycles of implantation and decay periods. The proton beam was pulsed during the implantation part of the cycles. Laser-off runs were taken for the setting on ${ }^{72} \mathrm{Ni}$ only. More details on the production methods and the detection setups used for the $\beta$-decay study of neutron-rich isotopes at the LISOL and ISOLDE facilities can be found in $[5,19]$ and $[7,20]$, respectively.

\section{B. Analysis procedure}

For each setting, both $\beta$-conditioned and nonconditioned $\gamma$ spectra were taken. The comparison between the two sets of data was made to identify $\gamma$ rays originating from the environment (background lines) or associated with the decay of long-lived contaminants (contamination lines). The latter contribution had different origins, depending on the installation where the experiment was performed: For the study of ${ }^{72} \mathrm{Cu}$ at ISOLDE, the contaminating activity was related to the implantation of long-lived isotopes next to the tape during previous runs and from surface ionization of fission fragments inside the ion source. For the study of ${ }^{72} \mathrm{Ni}$ at LISOL, long-lived fission fragments extracted from the laser ionization source in a $2^{+}$charge state with the same $A / q$ ratio as the selected ion were also transmitted to the detection setup.

To reduce the activity from implanted contaminants, the tape was moved after a given number of cycles. As shown in Table I, the tape was moved every cycle of $7.5 \mathrm{~s}$ for ${ }^{72} \mathrm{Cu}$ and after 150 cycles $(90 \mathrm{~s})$ for the setting on ${ }^{72} \mathrm{Ni}$.

As reported in Table I, the production yield of $10 \mathrm{ions} / \mu \mathrm{C}$ measured at LISOL for ${ }^{72} \mathrm{Ni}$ was six orders of magnitude lower than the one obtained at ISOLDE for the production of ${ }^{72} \mathrm{Cu}$. As a consequence, the $\beta$-conditioned $\gamma$ data for the setting on ${ }^{72} \mathrm{Ni}$ are dominated by contamination lines. Laser-off runs were therefore performed to disentangle the $\gamma$ rays following the $\beta$ decay of ${ }^{72} \mathrm{Ni}$ (and of its short-lived daughter ${ }^{72} \mathrm{Cu}$ ) and the contamination lines.

Once the main $\gamma$ transitions have been identified in ${ }^{72} \mathrm{Zn}$ $\left({ }^{72} \mathrm{Cu} \beta\right.$ decay) and in ${ }^{72} \mathrm{Cu}\left({ }^{72} \mathrm{Ni} \beta\right.$ decay), $\gamma-\gamma$ coincidences were performed to detect low-intensity $\gamma$ rays and to place them in the $\beta$-decay scheme of the dedicated nucleus. 
The relative intensity of the observed $\gamma$ rays was derived from the $\beta$-gated $\gamma$ spectra. By considering the high energy of the $\beta$ particles emitted in the decay of ${ }^{72} \mathrm{Cu}\left(Q_{\beta}=8.3 \mathrm{MeV}\right.$ [21]) and ${ }^{72} \mathrm{Ni}\left(Q_{\beta}=5.8 \mathrm{MeV}\right.$ [21]) and the low detection threshold (about one hundred $\mathrm{keV}$ ) of the plastic $\Delta E$ detectors, the $\beta$-detection efficiency was taken as a constant in each experiment whatever the energy of the level fed. As a result, no energy-dependent correction was applied to the relative intensity of the $\gamma$ rays.

Standard $\gamma$ calibration sources do not allow us to determine with a good precision the $\gamma$-detection efficiency at energies above $2 \mathrm{MeV}$. However, the behavior of HPGe detectors can be reliably reproduced by means of Monte Carlo simulations [22]. The $\gamma$-detection efficiency was therefore derived in the 0 - to 4-MeV range for the two experiments from simulations using the GEANT code [23]. They were validated up to $1.4 \mathrm{MeV}$ using ${ }^{60} \mathrm{Co}$ and ${ }^{152} \mathrm{Eu}$ calibration sources. In case cascading $\gamma$ rays or cross-over transitions were observed in the same detector, the relative intensities were corrected to account for possible summing effects. ${ }^{1}$

\section{EXPERIMENTAL RESULTS}

\section{A. The $\beta$-decay study of ${ }^{72} \mathrm{Cu}$ at ISOLDE}

\section{1. $\gamma$-ray identification}

Representative single and $\beta$-gated coincidence spectra obtained in the decay of ${ }^{72} \mathrm{Cu}$ with the HPGe detector having the best resolution are shown in Fig. 1. The strongest $\gamma$ rays are listed in Table II. The peak areas, also shown in the table, were extracted from the $\beta$-gated $\gamma$ spectrum obtained for the full data set.

The time behavior of the identified $\gamma$ lines was checked in the following way: The counting rate obtained for each photo peak during the decay part of the cycles was fitted by means of a single-component exponential decay function after a background subtraction was applied. The $\gamma$-ray identification was refined by means of $\gamma-\gamma$ coincidences. For some $\gamma$ rays, there is only weak evidence for a real coincidence. Their peak areas are indicated with a question mark in Table II.

Three $\gamma$ rays at $145.1,352.4$, and $1461.1 \mathrm{keV}$ disappear when the $\beta$-coincident condition is applied. The latter two are well-known background lines originating from ${ }^{214} \mathrm{~Pb}$ and ${ }^{40} \mathrm{~K}$, respectively. The $145.1-\mathrm{keV} \gamma$ line was attributed to the $\beta-\gamma$ decay of ${ }^{72} \mathrm{Zn}\left(T_{1 / 2}=46.5 \mathrm{~h}, Q_{\beta}=458.4 \mathrm{keV}\right.$ [21]). The associated $\beta$ particles have a maximum energy of $458.4-$ $145.1=313.3 \mathrm{keV}$, and most of them may not have enough energy to trigger the $\beta$ detectors.

The time behavior of the $\gamma$ rays was fitted with a single exponential, revealing a half-life value reported in Table II. Most of the $\gamma$ rays agree with the ${ }^{72} \mathrm{Cu}$ lifetime of 6.6(1) s given in [10]. However, the fitting procedure gives negative half-life

\footnotetext{
${ }^{1}$ The probability that two cascading $\gamma$ rays contribute to the photo peak of the associated cross-over transition is equal to $\varepsilon_{\gamma 1} \times \varepsilon_{\gamma 2}$, where $\varepsilon_{\gamma 1,2}$ are the photo peak efficiencies of the cascading $\gamma$ rays.
}

values for the $\gamma$ rays at $264.6,487.6$, and $834.4 \mathrm{keV}$. This indicates that the half-life of their parent nucleus is probably much longer than the 7-s decay period of the cycles. The 264.6-keV $\gamma$ line does not show any clear coincidence with any other line and it was attributed to the decay of ${ }^{75} \mathrm{Ge}\left(T_{1 / 2}=\right.$ $82.78 \mathrm{~min}$ [21]). The $\gamma$ line at $487.6 \mathrm{keV}$ is in coincidence with the two lines at 386.5 and $619.4 \mathrm{keV}$, which are associated with long decay times. The three $\gamma$ rays were therefore attributed to the decay of ${ }^{71} \mathrm{Zn}^{m}\left(T_{1 / 2}=3.96 \mathrm{~h}\right.$ [21]). Both ${ }^{75} \mathrm{Ge}$ and ${ }^{71} \mathrm{Zn}$ may have been implanted next to the tape in previous settings. The $\gamma$ ray at $834.4 \mathrm{keV}$ is coincident with other $\gamma$ rays from the decay of ${ }^{72} \mathrm{Ga}\left(T_{1 / 2}=14.10 \mathrm{~h}\right.$ [21]). The latter is the daughter nucleus of ${ }^{72} \mathrm{Zn}$, originating from the $\beta$ decay of ${ }^{72} \mathrm{Cu}$ and directly produced from surface ionization in the ISOLDE ion source.

The remaining $55 \gamma$ lines were attributed to the decay of ${ }^{72} \mathrm{Cu}$ because they exhibit a short half-life or because they are coincident with known transitions in ${ }^{72} \mathrm{Zn}$. Furthermore, the $\gamma$ ray at $3355.0 \mathrm{keV}$ was considered as an escape peak from the intense $\gamma$ transition at $3865 \mathrm{keV}$, because of the energy and coincidence relations between the 511.00 - and $3355.0-\mathrm{keV}$ $\gamma$ rays.

\section{Decay scheme}

The decay scheme of ${ }^{72} \mathrm{Cu}$ was derived from the $\gamma-\gamma$ coincidence relations quoted in Table II. The result is shown in Fig. 2. Most of the $\gamma$ rays could be placed in the decay scheme unambiguously. Some weak lines, such as the 753.1, 928.6, and 1469.0-keV transitions, were placed on the basis of energy matching.

The relative $\gamma$-ray intensities were deduced from the area of the $\beta$-gated peaks listed in Table II, following the procedure described in Sec. II B. They are summarized in Table III and absolute intensities per $100 \beta$ decays are given in Fig. 2. These values were used to derive the $\beta$ branching ratios toward the deduced excited states in ${ }^{72} \mathrm{Zn}$.

In contrast to the previous $\beta$-decay study of ${ }^{72} \mathrm{Cu}$ by Runte et al. [10], no $\beta$-singles spectra where taken during the present experiment. As a consequence, the intensity of the direct $\beta$ feeding to the ground state of ${ }^{72} \mathrm{Zn}$ could not be inferred from a comparison between $\beta$-singles and $\beta$-gated $\gamma$ spectra. However, the ground-state spin of ${ }^{72} \mathrm{Cu}$ was assumed to be $I^{\pi}=\left(2^{ \pm}\right)$from a comparison between the decay schemes of ${ }^{70,72,74,76} \mathrm{Cu}$ (see next section) and from the decay study of ${ }^{72} \mathrm{Ni}$ (see Sec. III B). A direct feeding to the ${ }^{72} \mathrm{Zn}$ ground state would therefore involve a forbidden $2^{ \pm} \rightarrow 0^{+} \beta$ transition with $\Delta I=2$ and $\Delta \pi= \pm 1$. According to the compilation of Singh et al. [24], the corresponding $\log (f t)$ values are expected to be respectively larger than $10.6(\Delta \pi=+1)$ and $7.5(\Delta \pi=-1)$, depending on the parity of the ${ }^{72} \mathrm{Cu}$ ground state. Thus, the direct feeding to the ground state of ${ }^{72} \mathrm{Zn}$ would have a intensity weaker than $0.02 \%$ and $2.5 \%$, respectively. We assumed in the present work that it can be neglected.

The $\beta$ and $\gamma$-ray branching ratios shown in Fig. 2 are normalized to the total $\gamma$-decay strength obtained by summing the relative intensities of all the $\gamma$ rays decaying directly to the ground state of ${ }^{72} \mathrm{Zn}$. The reported $\log (f t)$ values were 

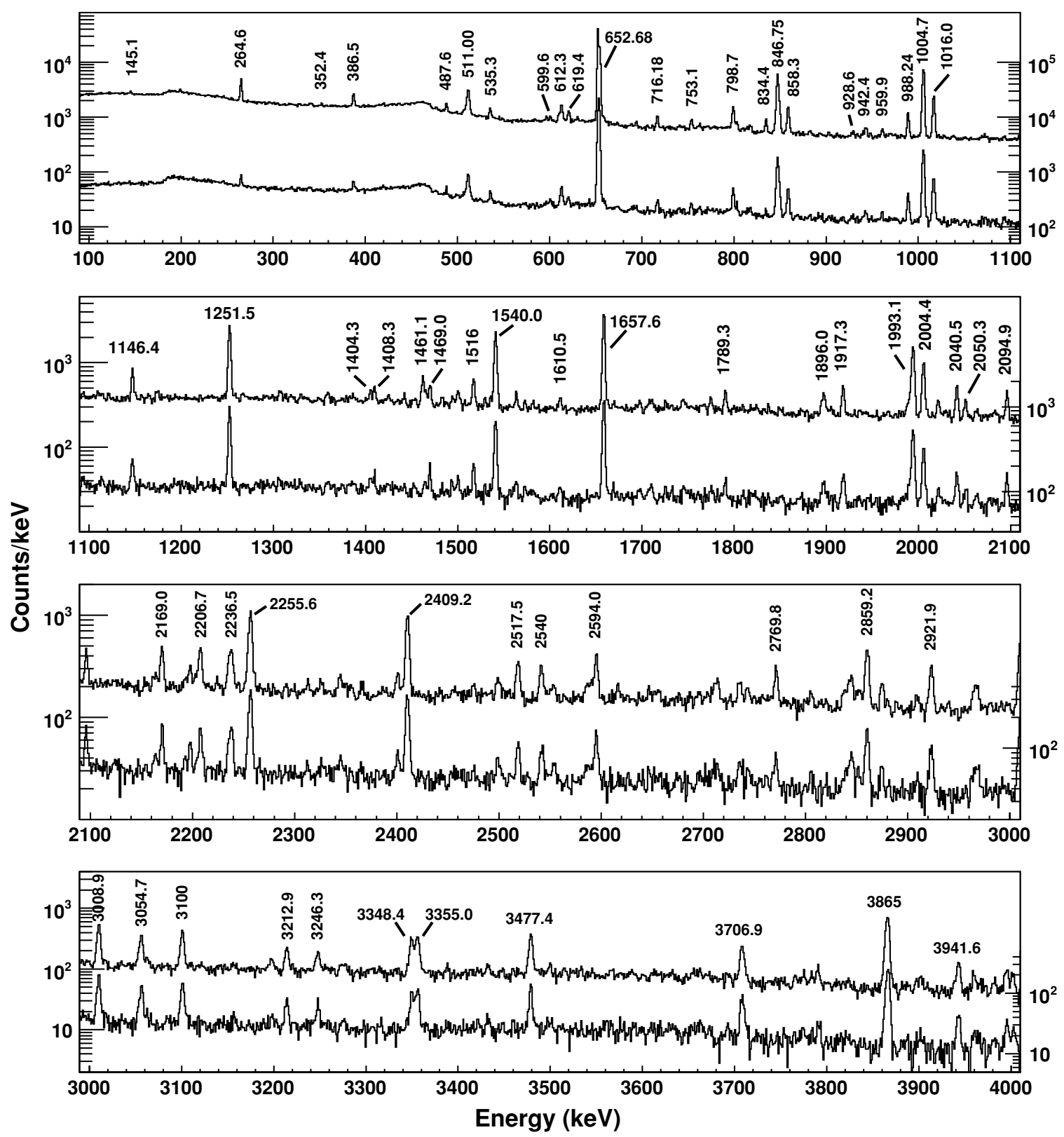

FIG. 1. Single (upper histogram, left scale) compared to $\beta$-gated coincident (lower histogram, right scale) $\gamma$ spectra obtained for the setting on ${ }^{72} \mathrm{Cu}$ with the HPGe detector having the best resolution. Histograms are represented with a binning of $1 \mathrm{keV} / \mathrm{channel}$. The energy of the $\gamma$ rays identified in the $\beta$ decay of ${ }^{72} \mathrm{Cu}$ is reported.

calculated by assuming no $\beta$ feeding to the ground state and have to be considered as lower limits. Levels identified in previous works [25] are marked with an x symbol in Fig. 2. The ${ }^{72} \mathrm{Cu}$ lifetime of 6.63(3) s was measured in the present work as described in Sec. III A4.

In general, the decay scheme proposed in Fig. 2 agrees well with the one of Runte et al. [10]. The decay scheme from the present work is more complete and it contains all the $\gamma$ transitions referenced in [10]. Only the 612.3- and 858.3-keV $\gamma$ transitions have a different placement: In [10], they link the excited states at 3662 and $2193 \mathrm{keV}$ via an intermediate state at $3050 \mathrm{keV}$. In the present work, a coincidence between the two $\gamma$ rays was excluded (see Table II). Hence, the intermediate excited state proposed in the work [10] at $3050 \mathrm{keV}$ is questionable.

\section{Spin and parity assignment}

In the previous $\beta$-decay study of ${ }^{72} \mathrm{Cu}$ by Runte $e t$ al. [10], the spin and parity of the excited states at 653,1499 , and $1658 \mathrm{keV}$ were adopted from the ${ }^{70} \mathrm{Zn}(t, p)^{72} \mathrm{Zn}$ reaction work of Hudson and Glover [11]. In the latter, excited states at $652(10), 1505(10)$, and $1652(10) \mathrm{keV}$ were observed and $I^{\pi}$ assignments of $2^{+}, 0^{+}$, and $2^{+}$were made by comparing the experimental $(t, p)$ cross sections with optical model 
TABLE II. Energy values, peak areas, and half-life values of the $\beta$-gated $\gamma$ lines identified in the decay of ${ }^{72} \mathrm{Cu}$. $\gamma$ lines with a negative value for $T_{1 / 2}$ were associated with the decay of parent nuclei with a half-life much longer than the fitting time interval. Coincident $\gamma$ rays are given in the last column of the table. Their peak areas are indicated within brackets and with a question mark when only weak evidence for a coincidence was found.

\begin{tabular}{|c|c|c|c|}
\hline$E(\mathrm{keV})$ & Area & $T_{1 / 2}(\mathrm{~s})$ & Coincident $\gamma$ rays \\
\hline 145.1(3) & - & - & - \\
\hline $264.6(1)$ & $900(100)$ & $-15(1)$ & - \\
\hline $386.5(1)$ & $1000(100)$ & $170(90)$ & $488[70], 511[30], 596[40], 619[70], 1107[20]$ \\
\hline $487.6(1)$ & $430(90)$ & $-6(2)$ & $387[60], 619[60]$ \\
\hline $511.00(6)$ & $3500(100)$ & $11(2)$ & $511[520], 653[70], 3355[40]$ \\
\hline $599.6(3)$ & $310(40)$ & - & $653[20], 1540[10]$ \\
\hline $612.3(1)$ & $1400(100)$ & $8(2)$ & $535[10], 653[130], 1540[30]$ \\
\hline $619.4(2)$ & $300(70)$ & $400(3500)$ & $387[60], 488[60]$ \\
\hline $652.68(5)$ & $89700(300)$ & $8.2(1)$ & $\begin{array}{c}600[20], 612[120], 847[770], 858[190], 988[50], 1005[1180], 1016[240], 1252[170], \\
1408[40], 1469[40], 1516[50], 1540[220], 1993[240], 2004[50], 2041[100], \\
2207[60], 2256[200], 2409[200], 2540[50], 2594[30], 2922[50], 3009[130], \\
3055[60], 3100[100], 3213[50], 3348[60], 3477[80], 3942[20]\end{array}$ \\
\hline $834.4(2)$ & $380(60)$ & $-40(90)$ & $630[20], 894[10], 2202[20]$ \\
\hline $846.75(7)$ & $8400(100)$ & $9.1(7)$ & $653[710], 942[20], 1146[30], 1896[20], 2207[20], 2859[20]$ \\
\hline $858.3(1)$ & $1490(50)$ & $8(2)$ & $653[150]$ \\
\hline $928.6(7)$ & $160(40)$ & - & $653[10], 1005[?], 1658[?]$ \\
\hline $942.4(3)$ & $240(40)$ & $10(9)$ & $653[30], 847[20]$ \\
\hline $959.9(3)$ & $230(40)$ & $4(2)$ & $653[20]$ \\
\hline $988.24(9)$ & $1270(40)$ & $14(5)$ & $653[30], 1005[20], 1016[20], 1658[30]$ \\
\hline $1004.7(2)$ & $11200(100)$ & $8.4(5)$ & $535[30], 653[1080], 799[30], 988[50], 1252[100], 1917[10], 2004[50], 2095[20]$ \\
\hline $1016.0(1)$ & $3150(60)$ & $9(1)$ & $653[170], 847[20], 988[30], 1005[?], 1146[10], 1658[10], 1993[90]$ \\
\hline $1146.4(2)$ & $730(60)$ & - & $653[40], 847[30]$ \\
\hline $1251.5(1)$ & $4170(80)$ & $8.3(9)$ & $653[120], 799[50], 1005[110], 1658[80]$ \\
\hline $1657.6(2)$ & $5950(70)$ & $8.9(8)$ & $799[20], 988[20], 1252[100], 2004[50]$ \\
\hline $1789.3(3)$ & $260(30)$ & $7(5)$ & $653[30]$ \\
\hline $1896.0(6)$ & $310(30)$ & $8(6)$ & $653[20], 847[20]$ \\
\hline $1917.3(3)$ & $540(40)$ & $6(3)$ & $653[30], 1005[20], 1658[10]$ \\
\hline 1993.1(9) & $2700(60)$ & $9(1)$ & $653[240], 1016[80]$ \\
\hline $2004.4(3)$ & $1530(50)$ & $15(6)$ & $653[40], 1005[40], 1658[40]$ \\
\hline $2040.5(3)$ & $470(40)$ & $12(8)$ & $653[40], 1005[10]$ \\
\hline $2050.3(5)$ & $230(30)$ & $5(3)$ & $653[10], 1005[10], 1658[?]$ \\
\hline 2094.9(3) & $430(40)$ & $5(2)$ & $653[20], 1005[20], 1658[?]$ \\
\hline $2169.0(3)$ & $530(50)$ & $9(4)$ & - \\
\hline 2206.7(3) & $560(40)$ & $7(2)$ & $653[30], 847[20], 1005[?]$ \\
\hline $2236.5(3)$ & $830(40)$ & $9(3)$ & $653[80], 1540[20]$ \\
\hline $2255.6(3)$ & $2050(60)$ & $7.1(9)$ & $653[130], 799[10]$ \\
\hline $2409.2(3)$ & $1780(50)$ & $9(2)$ & $653[200]$ \\
\hline $2517.5(4)$ & $360(30)$ & $9(6)$ & $653[20], 1005[10], 1658[?]$ \\
\hline $2540(2)$ & $330(30)$ & - & $653[50]$ \\
\hline $2594.0(4)$ & $500(40)$ & $8(3)$ & $653[30]$ \\
\hline $2769.8(4)$ & $290(30)$ & $7(4)$ & $653[10], 1005[10], 1658[10]$ \\
\hline $2859.2(4)$ & $770(40)$ & $8(3)$ & $847[40]$ \\
\hline
\end{tabular}


TABLE II. (Continued.)

\begin{tabular}{lrrc}
\hline \hline$E(\mathrm{keV})$ & \multicolumn{1}{c}{ Area } & $T_{1 / 2}(\mathrm{~s})$ & Coincident $\gamma$ rays \\
\hline $2921.9(5)$ & $450(30)$ & $10(6)$ & $653[50]$ \\
$3008.9(5)$ & $1050(40)$ & $8(2)$ & $653[140]$ \\
$3054.7(5)$ & $650(40)$ & $4.4(8)$ & $653[50]$ \\
$3100(2)$ & $820(40)$ & $8(3)$ & $653[90]$ \\
$3212.9(5)$ & $360(40)$ & $10(8)$ & $653[40]$ \\
$3246.3(6)$ & $200(40)$ & $6(3)$ & - \\
$3348.4(6)$ & $690(30)$ & $6(1)$ & $653[100]$ \\
$3355.0(7)$ & $700(30)$ & $7(2)$ & $511[40], 653[20]$ \\
$3477.4(6)$ & $620(40)$ & $7(2)$ & $653[60]$ \\
$3706.9(7)$ & $490(30)$ & $12(8)$ & - \\
$3865(1)$ & $1900(30)$ & $8(1)$ & $653[?]$ \\
$3941.6(7)$ & $250(30)$ & $3.3(7)$ & $653[20]$ \\
\hline \hline
\end{tabular}

calculations. Similarly, we will adopt the $I^{\pi}=2^{+}$assignment for both the 653- and $1658-\mathrm{keV}$ states. However, it is unclear whether the $0^{+}$state at $1505(10) \mathrm{keV}$ from Hudson and Glover corresponds to the 1499-keV state, as Runte et al. assumed, or to the state at $1511 \mathrm{keV}$ additionally observed in the present work. The answer is suggested by recent results obtained in multinucleon transfer reactions by Wilson et al. [12]. In this work, a $\left(4^{+}\right)$state at $1500 \mathrm{keV}$ is reported, which decays further down to the ground state of ${ }^{72} \mathrm{Zn}$ via two cascading $\gamma$ rays of 847.1 and $653.0 \mathrm{keV}^{2}$ They suggest this state to be

\footnotetext{
${ }^{2}$ In multinucleon transfer reactions, yrast excited states are more likely produced (i.e., the higher the excitation energy of a state is, the higher its spin is). Since the $1500-\mathrm{keV}$ state in [12] decays to the $2^{+}$ state at $653 \mathrm{keV}$, an $I^{\pi}$ value of $4^{+}$is more likely than $I^{\pi}=0^{+}$.
}

TABLE III. Relative intensities ( $\left.I_{\text {rel }}\right)$ of the $\gamma$ rays identified in the $\beta$ decay of ${ }^{72} \mathrm{Cu}$. Values are normalized with respect to the intensity of the transition at $652.68 \mathrm{keV}$. For absolute intensities per 100 $\beta$ decays, $I_{\text {rel }}$ has to be multiplied by 0.79 .

\begin{tabular}{lrlr}
\hline \hline$E(\mathrm{keV})$ & $I_{\text {rel }}$ & $E(\mathrm{keV})$ & $I_{\text {rel }}$ \\
\hline $535.3(2)$ & $0.64(6)$ & $1993.1(9)$ & $7.0(4)$ \\
$599.6(3)$ & $0.32(5)$ & $2004.4(3)$ & $4.0(2)$ \\
$612.3(1)$ & $1.5(1)$ & $2040.5(3)$ & $1.3(1)$ \\
$652.68(5)$ & $100(5)$ & $2050.3(5)$ & $0.55(9)$ \\
$716.18(7)$ & $0.73(8)$ & $2094.9(3)$ & $1.2(1)$ \\
$753.1(2)$ & $0.43(6)$ & $2169.0(3)$ & $1.5(1)$ \\
$798.7(1)$ & $1.8(1)$ & $2206.7(3)$ & $1.6(1)$ \\
$846.75(7)$ & $11.4(6)$ & $2236.5(3)$ & $2.4(2)$ \\
$858.3(1)$ & $2.0(1)$ & $2255.6(3)$ & $5.6(3)$ \\
$928.6(7)$ & $0.23(5)$ & $2409.2(3)$ & $5.6(3)$ \\
$942.4(3)$ & $0.35(6)$ & $2517.5(4)$ & $1.2(1)$ \\
$959.9(3)$ & $0.34(6)$ & $2540(2)$ & $1.1(1)$ \\
$988.24(9)$ & $1.9(1)$ & $2594.0(4)$ & $1.7(2)$ \\
$1004.7(2)$ & $17.3(9)$ & $2769.8(4)$ & $1.1(1)$ \\
$1016.0(1)$ & $4.9(3)$ & $2859.2(4)$ & $2.9(2)$ \\
$1146.4(2)$ & $1.2(1)$ & $2921.9(5)$ & $1.6(2)$ \\
$1251.5(1)$ & $7.6(4)$ & $3008.9(5)$ & $3.6(2)$ \\
$1404.3(5)$ & $0.38(6)$ & $3054.7(5)$ & $2.3(2)$ \\
$1408.3(3)$ & $0.74(9)$ & $3100(2)$ & $3.3(2)$ \\
$1469.0(2)$ & $0.71(7)$ & $3212.9(5)$ & $1.5(2)$ \\
$1516(2)$ & $1.1(1)$ & $3246.3(6)$ & $0.7(2)$ \\
$1540.0(2)$ & $6.8(4)$ & $3348.4(6)$ & $3.1(2)$ \\
$1610.5(5)$ & $0.31(6)$ & $3477.4(6)$ & $2.9(2)$ \\
$1657.6(2)$ & $11.7(6)$ & $3706.9(7)$ & $2.2(2)$ \\
$1789.3(3)$ & $0.59(9)$ & $3865(1)$ & $9.9(5)$ \\
$1896.0(6)$ & $0.80(9)$ & $3941.6(7)$ & $1.4(2)$ \\
$1917.3(3)$ & $1.4(1)$ & & \\
\hline \hline & & & \\
\hline
\end{tabular}




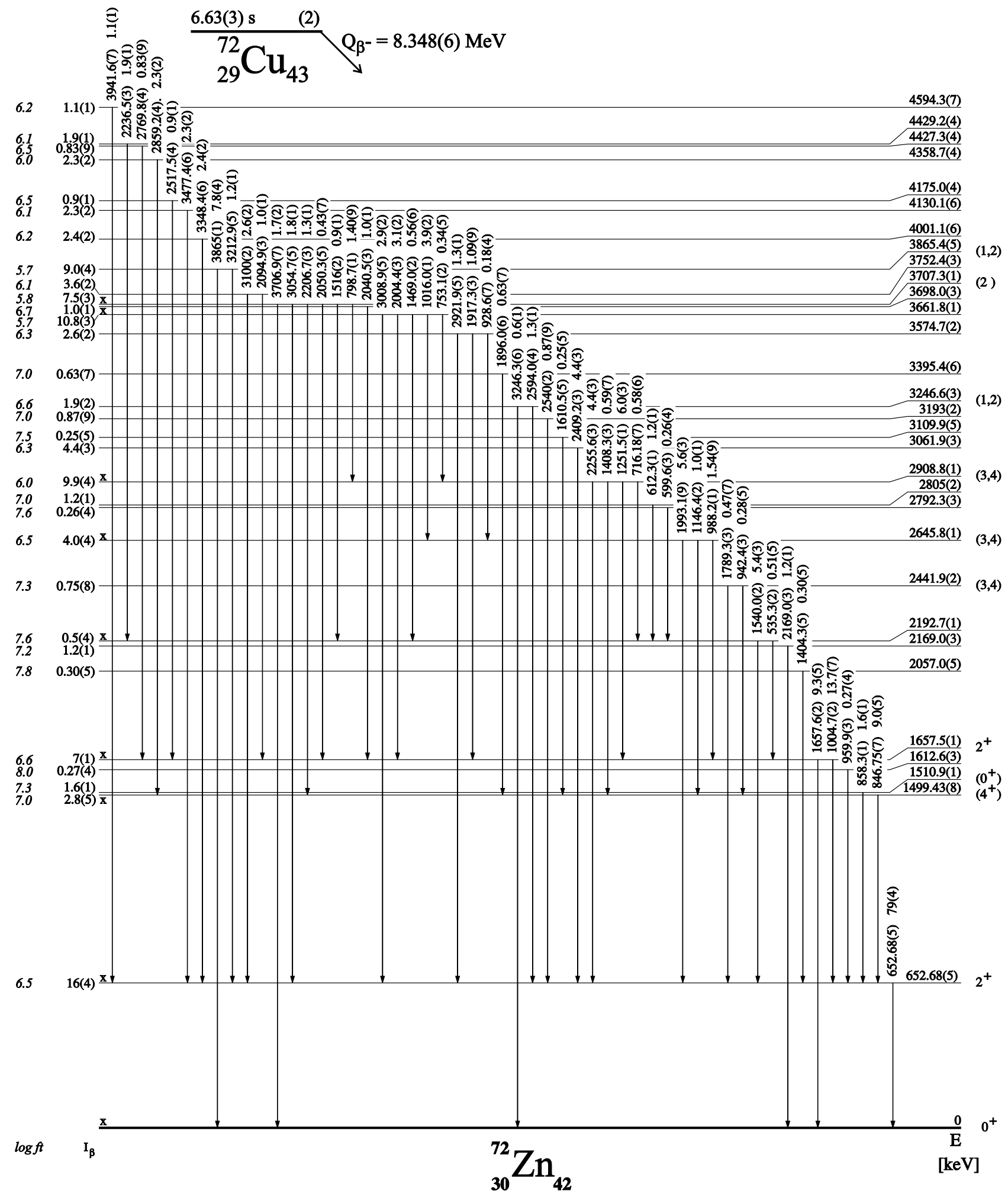

FIG. 2. Proposed level scheme for ${ }^{72} \mathrm{Zn}$. Levels labeled with " $\mathrm{x}$ " were observed in previous experiments [25].

different from the $1499-\mathrm{keV}$ state from Runte et al. because of the difference in $I^{\pi}$ and in energy $(\Delta E \sim 1 \mathrm{keV})$. Both states are finally assumed to be possible members of a two-phonon multiplet. However, with the presence of the newly determined state at $1511 \mathrm{keV}$ from this work, it is more likely that the
$I^{\pi}=4^{+}$state at $1500 \mathrm{keV}$ from [12] is the same than the $1499-\mathrm{keV}$ state reported in [10] and that the $1511-\mathrm{keV}$ state is the $0^{+}$state observed by Hudson and Glover. Hence, we propose a $I^{\pi}=\left(4^{+}\right)$assignment for the $1499-\mathrm{keV}$ excited state and $I^{\pi}=\left(0^{+}\right)$for the state at $1511 \mathrm{keV}$. 
TABLE IV. Intensity ratio of the $\left(4_{1}^{+}\right) \rightarrow\left(2_{1}^{+}\right)$to $\left(2_{1}^{+}\right) \rightarrow 0_{\mathrm{g} . \mathrm{s} .}^{+}$ $\gamma$ transitions in ${ }^{70,72,74,76} \mathrm{Zn}$ observed in the $\beta$ decay of ${ }^{70,72,74,76} \mathrm{Cu}$.

\begin{tabular}{lcccc}
\hline \hline${ }^{72} \mathrm{Cu}$, g.s. & ${ }^{74} \mathrm{Cu},(2,3)$ & ${ }^{70} \mathrm{Cu},\left(3^{-}\right)$ & ${ }^{76} \mathrm{Cu},(3,4)$ & ${ }^{70} \mathrm{Cu},\left(6^{-}\right)$ \\
\hline $11.4(9) \%$ & $13.0(9) \%$ & $52(6) \%$ & $60(4) \%$ & $94.7(6) \%$ \\
\hline \hline
\end{tabular}

The spin and parity assumption of $\left(4^{+}\right)$for the $1499-\mathrm{keV}$ state also agrees well with the observed decay patterns in Fig. 2: The states at 2442, 2646, and $2909 \mathrm{keV}$ decay to the $2^{+}$state(s) at $653 \mathrm{keV}$ (and $1658 \mathrm{keV}$ ) and to the $1499-\mathrm{keV}$ state, but not to the $0^{+}$ground state of ${ }^{72} \mathrm{Zn}$. If the $1499-\mathrm{keV}$ state would be a $0^{+}$state, we would expect these states to decay to the ground state as well. The absence of the decay to the ground state is explained ${ }^{3}$ if the $1499-\mathrm{keV}$ state has a spin value $I^{\pi}=4^{+}$and if the 2442, 2646, and 2909-keV states have spin values of 3 or 4 .

Using the same argument indicates that the states at 3247 and $3865 \mathrm{keV}$ have possible spin values of 1 or 2 since they populate the $0^{+}$ground state of ${ }^{72} \mathrm{Zn}$ and the $2^{+}$state at $653 \mathrm{keV}$. The state at $3707 \mathrm{keV}$ is restricted to a spin value of (2) since it decays to the $0^{+}$ground state, to the two $2^{+}$states at 653 and $1658 \mathrm{keV}$, and to the $\left(4^{+}\right)$state at $1499 \mathrm{keV}$.

The largest amount of $\beta$-decay strength (57.2\%) feeds the states at 653, 1658, 2909, 3662, 3707, and $3865 \mathrm{keV}$ : Most of these states have spin ranging from (2) to (4). This suggests that the ground state of ${ }^{72} \mathrm{Cu}$ has a spin value ranging from (1) to (3). The spin value can be tentatively inferred from a comparison of the ground-state $\beta$ decays of neighboring even-even copper isotopes. No direct $\beta$ feeding nor $\gamma$ decay to the first $4^{+}$excited state of ${ }^{70} \mathrm{Zn}$ was observed in the $\beta$ decay of the $1^{+}$isomeric state of ${ }^{70} \mathrm{Cu}$ [7]. Hence, the feeding to the $\left(4^{+}\right)$excited state at $1499 \mathrm{keV}$ in ${ }^{72} \mathrm{Zn}$ observed in the present work suggests a spin $I>1$ for the ground state of ${ }^{72} \mathrm{Cu}$. Furthermore, Table IV shows that the intensity ratio of the $\left(4_{1}^{+}\right) \rightarrow\left(2_{1}^{+}\right)$to $\left(2_{1}^{+}\right) \rightarrow 0_{\text {g.s. }}^{+} \gamma$ transitions in ${ }^{72} \mathrm{Zn}$ agrees well with the one obtained in ${ }^{74} \mathrm{Zn}$ in the $\beta$ decay of the $I=(2,3)$ ground state of ${ }^{74} \mathrm{Cu}[8]$, whereas it disagrees with the yields measured in the decays of the $I^{\pi}=3^{-}$ isomeric state and $I^{\pi}=6^{-}$ground state of ${ }^{70} \mathrm{Cu}$ [7] and in the decay of the $I=(3,4)$ ground state of ${ }^{76} \mathrm{Cu}[8]$. A spin assignment $I=(2)$ is therefore favored for the ground state of ${ }^{72} \mathrm{Cu}$.

During an in-source laser spectroscopy experiment dedicated to neutron-rich $\mathrm{Cu}$ isotopes, three $\beta$-decaying isomers were observed in ${ }^{70} \mathrm{Cu}$ [7]. Using this technique for ${ }^{72} \mathrm{Cu}$, however, revealed no evidence for different $\beta$-decaying isomeric states.

\section{Lifetime evaluation}

The lifetime evaluation of ${ }^{72} \mathrm{Cu}$ was performed by using the time spectrum delivered by the $4 \pi \beta$ detector being part of the second detection setup. The daughter nucleus ${ }^{72} \mathrm{Zn}$ has a half-

\footnotetext{
${ }^{3}$ Here and in the following, the argument is based on the selection rule $\Delta I \leqslant 2$ (no $0 \rightarrow 0$ ) for fast $\gamma$ decays.
}

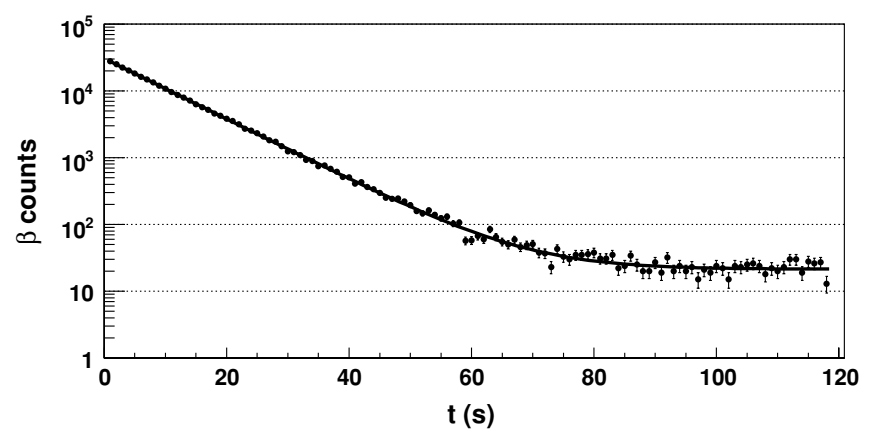

FIG. 3. Number of counts in the $\beta$ detector (dots) as a function of time. The solid line is the result of a fit of a single-component exponential decay function on a constant background.

life of $46.5 \mathrm{~h} \mathrm{[21]} \mathrm{and} \mathrm{its} \mathrm{contribution} \mathrm{to} \mathrm{the} \beta$-decay activity was considered as a constant background in view of the time scale used. The same holds for possible surface ionized ${ }^{72} \mathrm{Ga}$ $\left(T_{1 / 2}=14.10 \mathrm{~h}[21]\right)$. Thus, the ${ }^{72} \mathrm{Cu} \beta$-decay time spectrum was fitted with a single-component exponential decay function added to a constant background. The fit of the spectrum is shown by the solid line in Fig. 3. A half-life of 6.63(3) s was obtained for ${ }^{72} \mathrm{Cu}$, in perfect agreement with the value of 6.6(1) s reported in [10].

The influence of the dead time of the data-acquisition system was investigated by starting the fit of the time spectrum at different positions. No significant change in the derived lifetime value was observed.

\section{B. The $\beta$-decay study of ${ }^{72} \mathrm{Ni}$ at LISOL}

\section{1. $\gamma$-ray identification}

Figure 4 shows a superposition of the $\beta$-gated $\gamma$ spectra obtained in the decay of ${ }^{72} \mathrm{Ni}$ with (upper histogram) and without (lower histogram) selective laser ionization of $\mathrm{Ni}$ atoms. Because the beam time devoted to laser-off runs was quite short (see Table I), the corresponding spectrum in Fig. 4 was normalized by a factor of 16 for comparison with the laser-on data. For visibility, the laser-on spectrum is plotted with an additional offset of 3000 counts per channel in the top part of the picture, of 500 counts per channel in the middle part, and of 200 counts per channel in the bottom part.

Nonresonant $\gamma$ lines were attributed to the decay of ${ }^{144} \mathrm{Ba}\left(T_{1 / 2}=11.5 \mathrm{~s}[21]\right)$ and of its daughter nucleus ${ }^{144} \mathrm{La}$ $\left(T_{1 / 2}=40.8 \mathrm{~s}\right.$ [21]). As shown in Fig. 4, the most intense $\gamma$ rays following their $\beta$ decay are visible in the laser-on as well as in the laser-off spectra. Owing to their relatively short lifetimes, most of their activity was observed before the implantation tape was moved after each cycle of $90 \mathrm{~s}$ (Table I). ${ }^{144} \mathrm{Ba}$ is strongly produced in the fission of ${ }^{238} \mathrm{U}$ and is partly extracted from the laser ion source in a $2^{+}$charge state. The mass resolution $M / \Delta M \approx 1000$ of the LISOL mass separator does not allow filtering of this contamination since the $A / Q$ values of ${ }^{72} \mathrm{Ni}$ and ${ }^{144} \mathrm{Ba}$ are too close to each other: $\left[M\left({ }^{144} \mathrm{Ba}\right) / 2-M\left({ }^{72} \mathrm{Ni}\right)\right] / M\left({ }^{72} \mathrm{Ni}\right) \approx 3 . \times 10^{-4} .4$ The

\footnotetext{
${ }^{4}$ Atomic masses were taken from [26].
} 

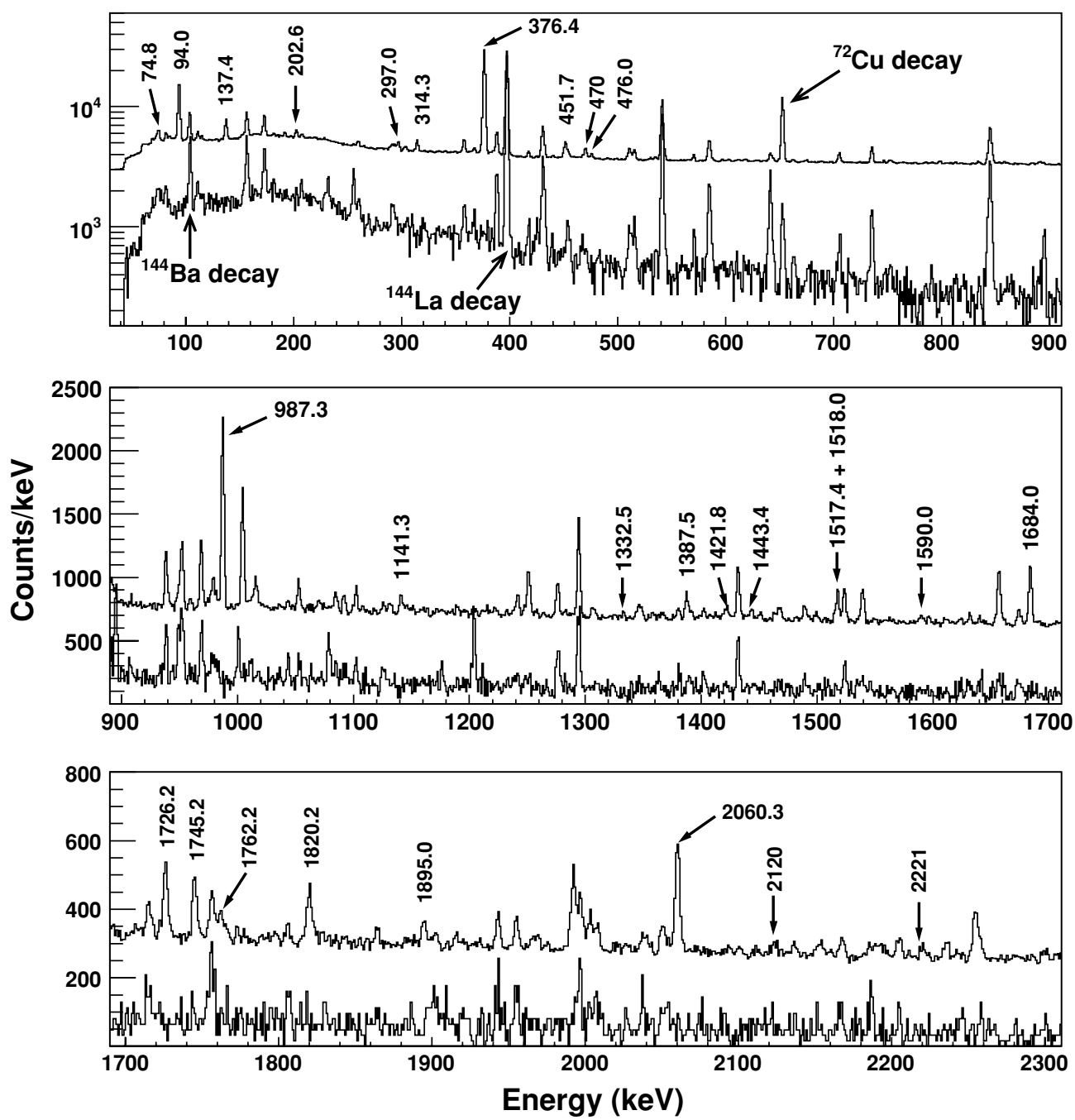

FIG. 4. $\beta$-gated laser-on (upper histogram) and normalized laser-off (lower histogram) spectra obtained in the setting on ${ }^{72} \mathrm{Ni}$. Offsets of 3000,500 , and 200 counts per channel were added to the laser-on data displayed on the top, middle, and bottom parts of the picture, respectively. Histograms are represented with a binning of $1 \mathrm{keV} /$ channel. The energy of the $\gamma$ rays identified in the $\beta$ decay of ${ }^{72} \mathrm{Ni}$ is reported.

activity of ${ }^{144} \mathrm{La}$ is supposed to originate from the $\beta$ decay of ${ }^{144} \mathrm{Ba}$ and not from a direct contamination. Its second ionization potential is indeed higher than the one of ${ }^{144} \mathrm{Ba}$ and it is presumably it does not significantly survive in a $2^{+}$ charge state inside the laser ion source [27].

The resonant $\gamma$ lines were attributed to the decay of ${ }^{72} \mathrm{Ni}$, unless their energy and intensity matched the ones of the $\gamma$ lines previously identified in the decay of ${ }^{72} \mathrm{Cu}$. They are listed in Table $\mathrm{V}$ with their associated areas. The peaks at $1332.5,1421.8$, and $1518.0 \mathrm{keV}$ could not be identified by comparing the $\beta$-gated laser-on and laser-off $\gamma$ spectra. They were attributed to the $\beta-\gamma$ decay of ${ }^{72} \mathrm{Ni}$ on the basis of $\gamma-\gamma$ coincidence relations: As illustrated in Fig. 5, the $\gamma$ ray at $1332.5 \mathrm{keV}$ is clearly coincident with the one at $376.4 \mathrm{keV}$ whereas it is hardly noticeable in the $\beta$-gated laser on spectrum of Fig. 4.

Many of the $\gamma$ transitions associated with the decay of ${ }^{72} \mathrm{Ni}$ are contaminated by low-intensity $\gamma$ rays originating from the $\beta$ decay of ${ }^{72} \mathrm{Cu},{ }^{144} \mathrm{Ba}$, and ${ }^{144} \mathrm{La}$. As a result, the area of the peaks of interest was most often corrected for the expected contribution of the contamination lines. The peak areas corrected at a level of more than $10 \%$ are quoted with a asterisk in Table $\mathrm{V}$. The main consequence of this correction procedure is that large uncertainties were obtained for the areas of some low-intensity $\gamma$ rays.

In Fig. 4, the peak at $470 \mathrm{keV}$ was found to originate from true summing of the intense coincident $\gamma$ transitions at 94 and $376 \mathrm{keV}$. It is therefore not reported in Table V. True summing corrections of less than $20 \%$ were also applied to a few other $\gamma$ rays.

\section{Decay scheme}

The decay scheme of ${ }^{72} \mathrm{Ni}$ is proposed in Fig. 6. It was derived from the coincidence relations between the identified $\gamma$ lines. As shown in Table $\mathrm{V}$ and in Fig. 5, half of the $\gamma$ transitions are coincident with the one at $376.4 \mathrm{keV}$. They were placed in the decay scheme with respect to their energy, relative intensity, and coincidence relation between each other. As shown in Fig. 6, nine transitions were found to feed directly 
TABLE V. Energy values, peak areas, and coincident $\gamma$ rays (which peak areas are shown in brackets) of the $\gamma$ lines identified in the $\beta$ decay of ${ }^{72} \mathrm{Ni}$. Peak areas that were corrected more than $10 \%$ for the contribution of the ${ }^{144} \mathrm{Ba}$ and ${ }^{144} \mathrm{La}$ contaminants or for the $\beta$ decay of the daughter nucleus ${ }^{72} \mathrm{Cu}$ are marked with an asterisk $\left(^{*}\right)$.

\begin{tabular}{|c|c|c|}
\hline$E(\mathrm{keV})$ & Area & Coincident $\gamma$ rays \\
\hline $74.8(1)$ & $800(100)$ & $376[260]$ \\
\hline $94.0(1)$ & $25900(200)$ & $\begin{array}{c}203[400], 376[5870], \\
1388[50], \\
1590[50], 1726[110]\end{array}$ \\
\hline $137.4(1)$ & $5900(500)^{*}$ & $314[950], 1443[30], 1745[70]$ \\
\hline $202.6(1)$ & $2000(300)^{*}$ & $\begin{array}{c}94[370], 376[280], 470[70], \\
1388[40]\end{array}$ \\
\hline 297.0(1) & $1600(400)^{*}$ & $376[450], 1388[90]$ \\
\hline $314.3(1)$ & 2300(100) & $137[910], 1443[?], 1745[40]$ \\
\hline $376.4(1)$ & $62200(900)$ & $\begin{array}{c}75[190], 94[5600], 203[300], \\
297[470], 1141[100], \\
1333[40], \\
1388[140], 1518[140], \\
1590[50], \\
1684[390], 1726[160], \\
1820[100], \\
2221[40]\end{array}$ \\
\hline $451.7(1)$ & $2700(800)^{*}$ & $1443[30], 1745[60]$ \\
\hline $476.0(1)$ & $800(100)^{*}$ & $1422[30]$ \\
\hline $987.3(1)$ & $4000(200)$ & $\begin{array}{c}653[20], 1004[30], 1016[10], \\
1654[10]\end{array}$ \\
\hline $1141.3(5)$ & $<590$ & $376[100]$ \\
\hline $1332.5(2)$ & $180(40)$ & $376[30]$ \\
\hline $1387.5(2)$ & $450(80)^{*}$ & $\begin{array}{c}94[40], 203[50], 297[100], \\
376[100]\end{array}$ \\
\hline $1421.8(9)$ & $<150^{*}$ & $476[30]$ \\
\hline $1443.4(9)$ & $>130$ & $\begin{array}{c}75[10], 137[30], 314[20], \\
452[30]\end{array}$ \\
\hline $1517.4(1)$ & $<320^{*}$ & \\
\hline $1518.0(2)$ & $550(60)$ & $376[130]$ \\
\hline $1590.0(1)$ & $<300$ & $94[40], 376[30]$ \\
\hline $1684.0(4)$ & $1300(100)^{*}$ & $376[380]$ \\
\hline $1726.2(1)$ & $<920$ & $94[140], 376[160], 470[20]$ \\
\hline 1745.2(3) & $630(50)$ & $\begin{array}{c}75[20], 137[90], 314[40] \\
376[30], 452[60]\end{array}$ \\
\hline $1762.2(2)$ & $400(200)^{*}$ & \\
\hline 1820.2(7) & $400(200)^{*}$ & $376[100]$ \\
\hline $1895.0(4)$ & $190(70)^{*}$ & \\
\hline $2060.3(1)$ & $1200(100)$ & \\
\hline $2120(1)$ & $60(20)$ & $476[20]$ \\
\hline 2221(1) & $160(60)$ & $376[30]$ \\
\hline
\end{tabular}

the ground state of ${ }^{72} \mathrm{Cu}$, with the one at $376.4 \mathrm{keV}$ accounting more than $70 \%$ of the total direct $\gamma$-decay strength.

The relative intensities of the $\gamma$ transitions are presented in Table VI. The large uncertainties of about $30 \%$ are due to the poor precision reached in determining experimentally the relative $\gamma$-detection efficiency and to the corrections applied to take into account the contribution to the photo peaks of $\gamma$ lines originating from the $\beta$ decay of ${ }^{144} \mathrm{Ba},{ }^{144} \mathrm{La}$, and ${ }^{72} \mathrm{Cu}$.

The $\beta$ branching ratios $I_{\beta}$ toward ${ }^{72} \mathrm{Cu}$ excited states as well as their associated $\log (f t)$ values are given in Fig. 6. They were

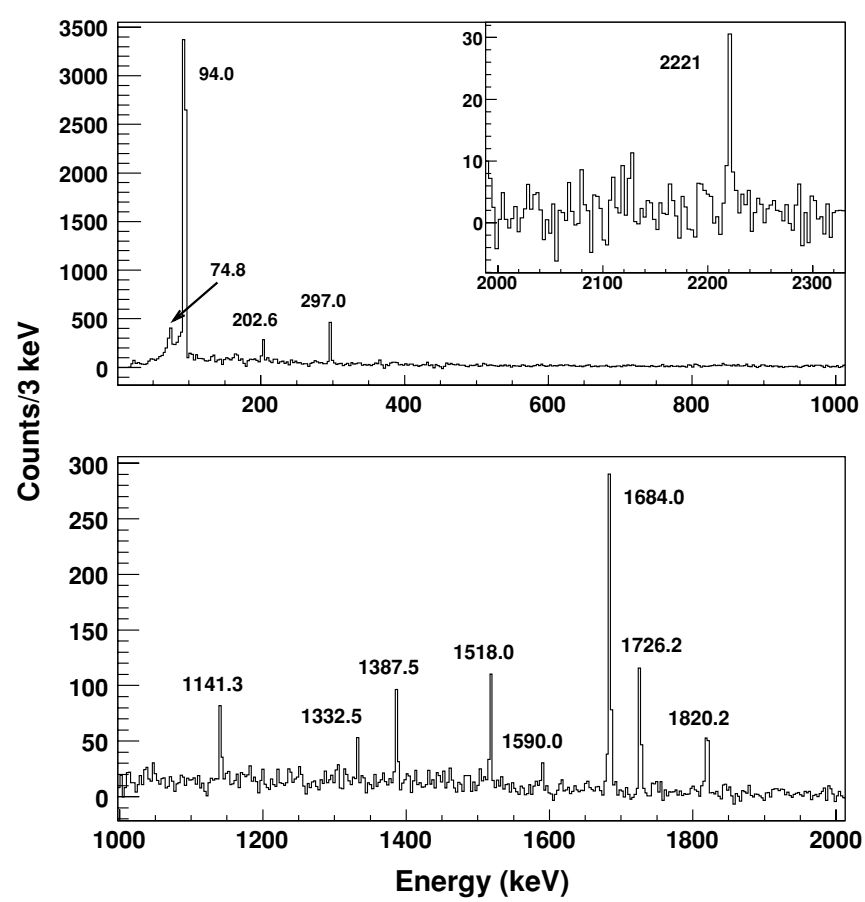

FIG. 5. $\gamma$ spectrum obtained for the setting on ${ }^{72} \mathrm{Ni}$ in coincidence with the $\beta$-conditioned $\gamma$ ray at $376.4 \mathrm{keV}$. The histogram is represented with a binning of $3 \mathrm{keV} /$ channel.

calculated by using the lifetime value of ${ }^{72} \mathrm{Ni}$ determined in the work [14] and assuming no direct feeding to the presumably $I=(2)$ ground state of ${ }^{72} \mathrm{Cu}$ : Such a decay from the $0^{+}$ground state of ${ }^{72} \mathrm{Ni}$ would indeed involve a forbidden $\beta$ transition with $\Delta I=2$ and $\Delta \pi= \pm 1$. According to the compilation of Singh et al. [24], the corresponding $\log (f t)$ values are expected to be larger than $10.6(\Delta \pi=+1)$ and $7.5(\Delta \pi=-1)$, respectively, which means that the direct feeding to the ${ }^{72} \mathrm{Cu}$ ground state is expected to have an absolute intensity lower than $\left(8 . \times 10^{-5}\right) \%$ or $0.1 \%$, depending on its parity.

The $\log (f t)$ values were corrected to take into account the contribution of the electronic conversion process to the electromagnetic decay of the lowest excited states. On the basis of spin and parity assignments discussed in the next section, the most probable multipolarities were adopted for the transitions at 74.8, 94.0, 137.4, 314.3, 376.4, and $451.7 \mathrm{keV}$. According to previous studies [16-18], none of the related decaying states is likely to be a long-lived isomer and we checked that the expected lifetimes associated with the adopted multipolarities were indeed shorter than 1 ps. The largest correction (13\%) concerned the very low intensity $\gamma$ line at $74.8 \mathrm{keV}$ and has therefore little influence on the evaluated $\beta$ branching ratio toward the excited state at $452 \mathrm{keV}$. However, a correction of $6 \%$ had to be applied to the transition at $94.0 \mathrm{keV}$ having a relative intensity of $27 \%$. This leads to an increase of the $\beta$ feeding to the excited state at $470 \mathrm{keV}$ of less than $10 \%$.

For comparison, the low-energy part of the level structure of ${ }^{72} \mathrm{Cu}$ obtained in the work of Mach et al. [17] is shown in the right part of Fig. 6 . In addition to the $\left(3^{-}\right)$excited state at $138 \mathrm{keV}$ observed as well in the present work, two other negative-parity states were reported at $219\left(4^{-}\right)$and $270 \mathrm{keV}$ 

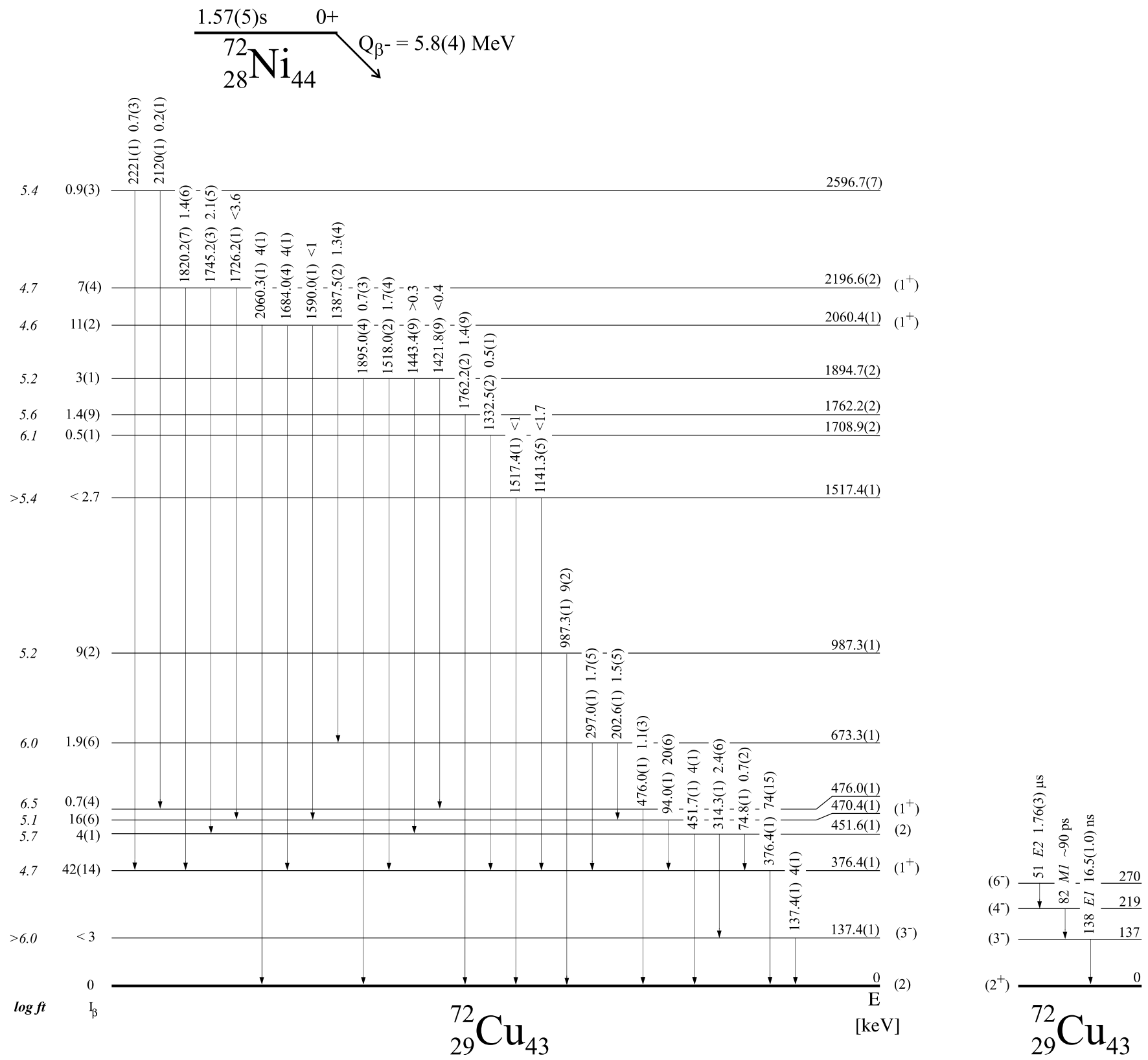

FIG. 6. Proposed level scheme for ${ }^{72} \mathrm{Cu}$. Spin and parity assignments were partly derived from a previous study [17,18]. Results from the latter work are presented in the right part of the figure.

$\left(6^{-}\right)$. The fact that they were not seen in the present work can be explained by the low spin and the positive parity of the $0^{+}$ground state of ${ }^{72} \mathrm{Ni}$. The same argument can be used to question the direct feeding to the $\left(3^{-}\right)$excited state at $137 \mathrm{keV}$. One may assume that there is actually no direct feeding to this state in the $\beta$ decay of ${ }^{72} \mathrm{Ni}$.

\section{Spin and parity assignment}

In the $\beta$-decay study of ${ }^{70} \mathrm{Ni}$ [7], the $0^{+}$ground state was found to decay preferably toward two $1^{+}$excited states at $242 \mathrm{keV}$ and $1278 \mathrm{keV}$ in ${ }^{70} \mathrm{Cu}$, with $\log (f t)$ values lower than 5. In the same way, a spin of $\left(1^{+}\right)$can be attributed to the excited states of ${ }^{72} \mathrm{Cu}$ at $376[\log (f t)=4.8], 2060$ $[\log (f t)=4.6]$, and $2197 \mathrm{keV}[\log (f t)=4.7]$. The excited state at $470 \mathrm{keV}$ has a slightly higher $\log (f t)$ value of 5.1 , but it only decays toward the $\left(1^{+}\right)$excited state at $376 \mathrm{keV}$. This behavior is closely related to that of the $\left(1^{+}\right)$excited state at $1278 \mathrm{keV}$ in ${ }^{70} \mathrm{Cu}$, decaying mainly toward the $1^{+}$excited state at $242 \mathrm{keV}$. The excited state at $470 \mathrm{keV}$ is therefore presumably a $\left(1^{+}\right)$state as well.

In the work [17], the ground-state spin of ${ }^{72} \mathrm{Cu}$ was assumed to be $I^{\pi}=\left(2^{+}\right)$, based on multipole and lifetime arguments. Considering the lifetime of the $138-\mathrm{keV}$ transition from the upper lying $\left(3^{-}\right)$state (see Fig. 6) gives its most probable multipolarity of $E 1, M 1$, or $E 2$, with an associated transition rate being, respectively, $10^{5}$ times slower, $2 . \times 10^{3}$ times slower, or 30 times faster than the corresponding Weisskopf 
TABLE VI. Relative intensities $\left(I_{\text {rel }}\right)$ of the $\gamma$ rays identified in the $\beta$ decay of ${ }^{72} \mathrm{Ni}$. Values are normalized with respect to the intensity of the transition at $376.4 \mathrm{keV}$. For absolute intensities per $100 \beta$ decays, $I_{\text {rel }}$ has to be multiplied by 0.74 .

\begin{tabular}{cc}
\hline \hline$E(\mathrm{keV})$ & $I_{\text {rel }}$ \\
\hline $74.8(1)$ & $0.9(3)$ \\
$94.0(1)$ & $27(7)$ \\
$137.4(1)$ & $5(2)$ \\
$202.6(1)$ & $2.1(6)$ \\
$297.0(1)$ & $2.2(6)$ \\
$314.3(1)$ & $3.3(6)$ \\
$376.4(1)$ & $100(16)$ \\
$451.7(1)$ & $5(2)$ \\
$476.0(1)$ & $1.4(3)$ \\
$987.3(1)$ & $12(2)$ \\
$1141.3(5)$ & $<2.2$ \\
$1332.5(2)$ & $0.7(2)$ \\
$1387.5(2)$ & $1.8(4)$ \\
$1421.8(9)$ & $<0.6$ \\
$1443.4(9)$ & $>0.5$ \\
$1517.4(1)$ & $<1.3$ \\
$1518.0(2)$ & $2.3(4)$ \\
$1590.0(1)$ & $<1.3$ \\
$1684.0(4)$ & $6(1)$ \\
$1726.2(1)$ & $<4.6$ \\
$1745.2(3)$ & $2.9(5)$ \\
$1762.2(2)$ & $2(1)$ \\
$1820.2(7)$ & $1.9(8)$ \\
$1895.0(4)$ & $0.9(4)$ \\
$2060.3(1)$ & $6(1)$ \\
$2120(1)$ & $0.3(1)$ \\
$2221(1)$ & $0.9(3)$ \\
\hline \hline & \\
\hline &
\end{tabular}

estimate. A systematic study of the pure electromagnetic transitions observed [28] in the 10 to $200-\mathrm{keV}$ range in the $62 \leqslant A \leqslant 82$ region reveals five pure $E 1$, two pure $M 1$, and eight pure $E 2$ transitions with transition rates, respectively, $5 . \times 10^{3}$ to $10^{5}$ times slower, $10^{2}$ to $10^{3}$ times slower, and 1 to 100 times faster than the associated Weisskopf estimates. An $E 2$ transition would result in a $\left(1^{-}\right)$or a $\left(5^{-}\right)$ground state for ${ }^{72} \mathrm{Cu}$. As shown in the next section, a $1^{-}$state cannot be obtained by considering the valence proton and neutron orbitals involved in ${ }^{72} \mathrm{Cu}$. A $5^{-}$ground state can be rejected as well since the intense transition at $376.4 \mathrm{keV}$ from the upper lying $\left(1^{+}\right)$state would be of $M 4$ character and therefore strongly hindered. However, an $E 1$ or an $M 1$ character of the transition at $137 \mathrm{keV}$ is consistent with observations in the same mass region. Thus, the spin of the ${ }^{72} \mathrm{Cu}$ ground state is presumably $I=(2)$, in agreement with the expected value derived earlier from its $\beta$-decay study.

Two $I=\left(2^{-}\right)$and $\left(2^{+}\right)$states were identified in the work [7] in the low-energy spectrum of ${ }^{70} \mathrm{Cu}$. They are fed in the $\gamma$ decay of two high-energy $\left(1^{+}\right)$states, which are mainly connected to lower lying $\left(1^{+}\right)$states. In the present work, the two $\left(1^{+}\right)$ states identified at 2060 and $2197 \mathrm{keV}$ are in the same way decaying preferably toward low-lying $\left(1^{+}\right)$states, but they are also decaying toward the $I=(2)$ ground state of ${ }^{72} \mathrm{Cu}$ and toward an excited state at $452 \mathrm{keV}$ (see Fig. 6). The latter decays toward the $I=(2)$ ground state of ${ }^{72} \mathrm{Cu}$, and toward the $I=\left(3^{-}\right)$and $I=\left(1^{+}\right)$excited states at 137 and $376 \mathrm{keV}$, respectively. We therefore assigned a spin $I=(2)$ to the state at $452 \mathrm{keV}$.

\section{DISCUSSION}

\section{A. Shell-model interpretation of the low-energy spectrum of ${ }^{72} \mathrm{Cu}$}

Shell-model calculations have been performed following two different approaches.

(i) First, we used a schematic model. We have considered the spectrum of the low-lying states in ${ }^{72} \mathrm{Cu}$ as originating from the coupling of the valence proton with a neutron quasi-particle beyond the ${ }^{70} \mathrm{Ni}$ core. Thus, the proton could occupy any of the four valence orbitals $\left(2 p_{3 / 2} 1 f_{5 / 2} 2 p_{1 / 2} 1 g_{9 / 2}\right)$, whereas neutrons were restricted to the $1 g_{9 / 2}$ orbital (where we will refer to $v 1 \tilde{g}_{9 / 2}$ as a neutron quasi-particle). As a result, only states of negative parity could be calculated. The interaction between the valence proton and neutrons was taken to be of $\delta$ type or a quadrupole-quadrupole one. The change of the single-proton energies reulting from the interaction with the $v 1 \tilde{g}_{9 / 2}$ neutrons (with ${ }^{68} \mathrm{Ni}$ being considered as a core) has been estimated via a monopole shift formula as described in [4]. All the details of this approach as well as the parameters of the interactions used can be found in [8].

(ii) In the second approach, the energy levels have been obtained via a large-scale shell-model diagonalization using the ANTOINE code [29] in the $\left(2 p_{3 / 2} 1 f_{5 / 2} 2 p_{1 / 2} 1 g_{9 / 2}\right)$ space outside the doubly magic ${ }_{28}^{56} \mathrm{Ni}_{28}$ core. We used a realistic effective interaction as derived by Hjorth-Jensen, Kuo, and Osnes [30] and modified further for the monopole part by Nowacki [31]. The interaction used in the present work differs from the one used in Refs. [4,6-8] by changes imposed onto three two-body centroids:

$$
\begin{aligned}
& \Delta V_{2 p_{3 / 2} 2 p_{3 / 2}}^{T=0}=-0.2 \mathrm{MeV}, \\
& \Delta V_{2 p_{1 / 2} 2 p_{1 / 2}}^{T=0}=-0.1 \mathrm{MeV}, \\
& \Delta V_{1 g_{9 / 2} 1 g_{9 / 2}}^{T=0}=-0.05 \mathrm{MeV} .
\end{aligned}
$$

In addition, some multipole modifications have been performed, which will be discussed in more detail elsewhere [32].

The resulting interaction gives a better agreement with the behavior of the lowest $5 / 2^{-}$state studied in neutron-rich odd-copper isotopes [4,5]. It also reduces the energy of the $\left(2_{1}^{-}\right)$state observed in ${ }^{70} \mathrm{Cu}$ at $369 \mathrm{keV}$ from $1275 \mathrm{keV}$ [7] to $755 \mathrm{keV}$.

To test further the interaction, the level scheme of ${ }^{72} \mathrm{Zn}$ was calculated with only four neutrons allowed to be excited from the $p f$ shell to the $1 g_{9 / 2}$ orbital. As shown in Fig. 7, the level scheme is in good agreement with the one obtained experimentally below $2 \mathrm{MeV}$ excitation energy, although the state observed at $1613 \mathrm{keV}$ is not reproduced. One can notice that the excitation energy of the $2_{1}^{+}$state drops experimentally by more than $200 \mathrm{keV}$ when going from ${ }^{70} \mathrm{Zn}$ [7] to ${ }^{72} \mathrm{Zn}$. The 

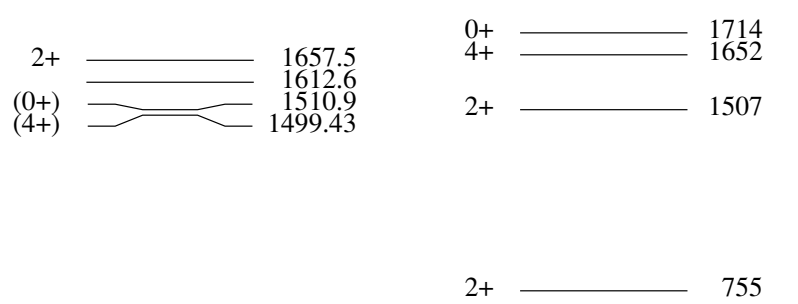

$2+\longrightarrow 652.68$

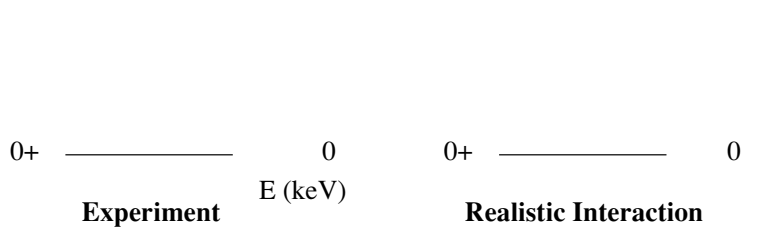

FIG. 7. Nuclear structure of ${ }^{72} \mathrm{Zn}$ below $2 \mathrm{MeV}$ of excitation energy. Nuclear states observed experimentally are compared to the theoretical spectrum calculated using the realistic interaction discussed in the text.

reason could be that the additional pair of neutrons occupying the $v 1 g_{9 / 2}$ orbital in ${ }^{72} \mathrm{Zn}$ somewhat weakens the $N=40$ subshell gap between the $v 2 p_{1 / 2}$ and the $\nu 1 g_{9 / 2}$ orbitals and can induce deformation.

Figure 8 compares the predictions of the different shellmodel approaches to the low-energy spectrum $\left(E^{*}<1 \mathrm{MeV}\right)$ of ${ }^{72} \mathrm{Cu}$ observed experimentally. The two spectra obtained with the schematic approach are shown in the right part of the figure. They consist in two (3-6) ${ }^{-}$and (2-7) ${ }^{-}$multiplets of $\pi 2 p_{3 / 2} \nu 1 \tilde{g}_{9 / 2}$ and $\pi 1 f_{5 / 2} \nu 1 \tilde{g}_{9 / 2}$ configurations, respectively, almost without any mixing between them. The low-energy positive-parity states, expected from the coupling of a proton in $\pi 2 p_{3 / 2}$ and a neutron hole in $v 2 p_{1 / 2}^{-1}$, are outside the model space.

The results of the large-scale diagonalization using the realistic interaction are shown next to the experimental level
TABLE VII. Nuclear structure of ${ }^{72} \mathrm{Cu}$ at low excitation energy. Nuclear states observed experimentally are compared to those derived from a large-scale shell-model calculation using a realistic effective interaction (see text for details). The ${ }^{*}$ symbols refer to states observed in [17]. For the $5^{-}$and $2_{2}^{+}$states only theoretical calculations exist.

\begin{tabular}{|c|c|c|c|c|}
\hline$I^{\pi}$ & $E_{\text {exp }}(\mathrm{keV})$ & $E_{\text {theor }}(\mathrm{keV})$ & Configuration & $(\%)$ \\
\hline$\left(2^{+}\right)$ & 0 or 451.6 & 431 & $\begin{array}{l}\pi 2 p_{3 / 2} v\left(2 p_{1 / 2}^{-1} 1 g_{9 / 2}^{4}\right) \\
\pi 2 p_{3 / 2} \nu\left(2 p_{3 / 2}^{-1} 1 g_{9 / 2}^{4}\right)\end{array}$ & $\begin{array}{l}(47) \\
(10)\end{array}$ \\
\hline$\left(3^{-}\right)$ & 137.4 & 0 & $\begin{array}{c}\pi 2 p_{3 / 2} \nu 1 g_{9 / 2}^{3} \\
\pi 2 p_{3 / 2} \nu\left(1 f_{5 / 2}^{-2} 1 g_{9 / 2}^{5}\right)\end{array}$ & $\begin{array}{l}(56) \\
(10)\end{array}$ \\
\hline$\left(4^{-}\right)^{*}$ & 219 & 140 & $\begin{array}{c}\pi 2 p_{3 / 2} \nu 1 g_{9 / 2}^{3} \\
\pi 2 p_{3 / 2} v\left(1 f_{5 / 2}^{-2} 1 g_{9 / 2}^{5}\right)\end{array}$ & $\begin{array}{l}(56) \\
(10)\end{array}$ \\
\hline$\left(6^{-}\right)^{*}$ & 270 & 16 & $\begin{array}{c}\pi 2 p_{3 / 2} \nu 1 g_{9 / 2}^{3} \\
\pi 2 p_{3 / 2} v\left(1 f_{5 / 2}^{-2} 1 g_{9 / 2}^{5}\right)\end{array}$ & $\begin{array}{l}(64) \\
(11)\end{array}$ \\
\hline $5^{-}$ & & 235 & $\begin{array}{c}\pi 2 p_{3 / 2} \nu 1 g_{9 / 2}^{3} \\
\pi 2 p_{3 / 2} v\left(1 f_{5 / 2}^{-2} 1 g_{9 / 2}^{5}\right)\end{array}$ & $\begin{array}{l}(62) \\
(11)\end{array}$ \\
\hline$\left(1_{1}^{+}\right)$ & 376.4 & 418 & $\begin{array}{l}\pi 2 p_{3 / 2} v\left(2 p_{1 / 2}^{-1} 1 g_{9 / 2}^{4}\right) \\
\pi 2 p_{1 / 2} v\left(2 p_{1 / 2}^{-1} 1 g_{9 / 2}^{4}\right)\end{array}$ & $\begin{array}{l}(45) \\
(10)\end{array}$ \\
\hline$\left(2^{-}\right)$ & 0 or 451.6 & 387 & $\begin{array}{l}\pi 1 f_{5 / 2} \nu 1 g_{9 / 2}^{3} \\
\pi 2 p_{3 / 2} \nu 1 g_{9 / 2}^{3}\end{array}$ & $\begin{array}{l}(33) \\
(19)\end{array}$ \\
\hline$\left(1_{2}^{+}\right)$ & 470.4 & 585 & $\begin{array}{l}\pi 2 p_{1 / 2} v\left(2 p_{1 / 2}^{-1} 1 g_{9 / 2}^{4}\right) \\
\pi 2 p_{3 / 2} v\left(2 p_{1 / 2}^{-1} 1 g_{9 / 2}^{4}\right) \\
\pi 1 f_{5 / 2} v\left(2 p_{1 / 2}^{-1} 1 g_{9 / 2}^{4}\right)\end{array}$ & $\begin{array}{l}(29) \\
(20) \\
(15)\end{array}$ \\
\hline $2_{2}^{+}$ & & 772 & $\begin{array}{l}\pi 1 f_{5 / 2} v\left(2 p_{1 / 2}^{-1} 1 g_{9 / 2}^{4}\right) \\
\pi 1 f_{5 / 2} \nu\left(2 p_{3 / 2}^{-1} 1 g_{9 / 2}^{4}\right)\end{array}$ & $\begin{array}{l}(48) \\
(10)\end{array}$ \\
\hline
\end{tabular}

scheme. Table VII gives the $\pi-v$ configurations derived from the large-scale shell-model calculation and contributing more than $10 \%$ to the lowest excited states observed experimentally. The low-energy spectrum of ${ }^{72} \mathrm{Cu}$ is found to be dominated by the $\pi 2 p_{3 / 2} \nu 1 g_{9 / 2}^{3}$ and $\pi 2 p_{3 / 2} \nu\left(2 p_{1 / 2}^{-1} 1 g_{9 / 2}^{4}\right)$ configurations, leading to the $(3-6)^{-}$and $(1,2)^{+}$multiplets of states, respectively. The expected $(2-7)^{-}$multiplet of the $\pi 1 f_{5 / 2} \nu 1 g_{9 / 2}^{3}$ dominant configuration is also reproduced.
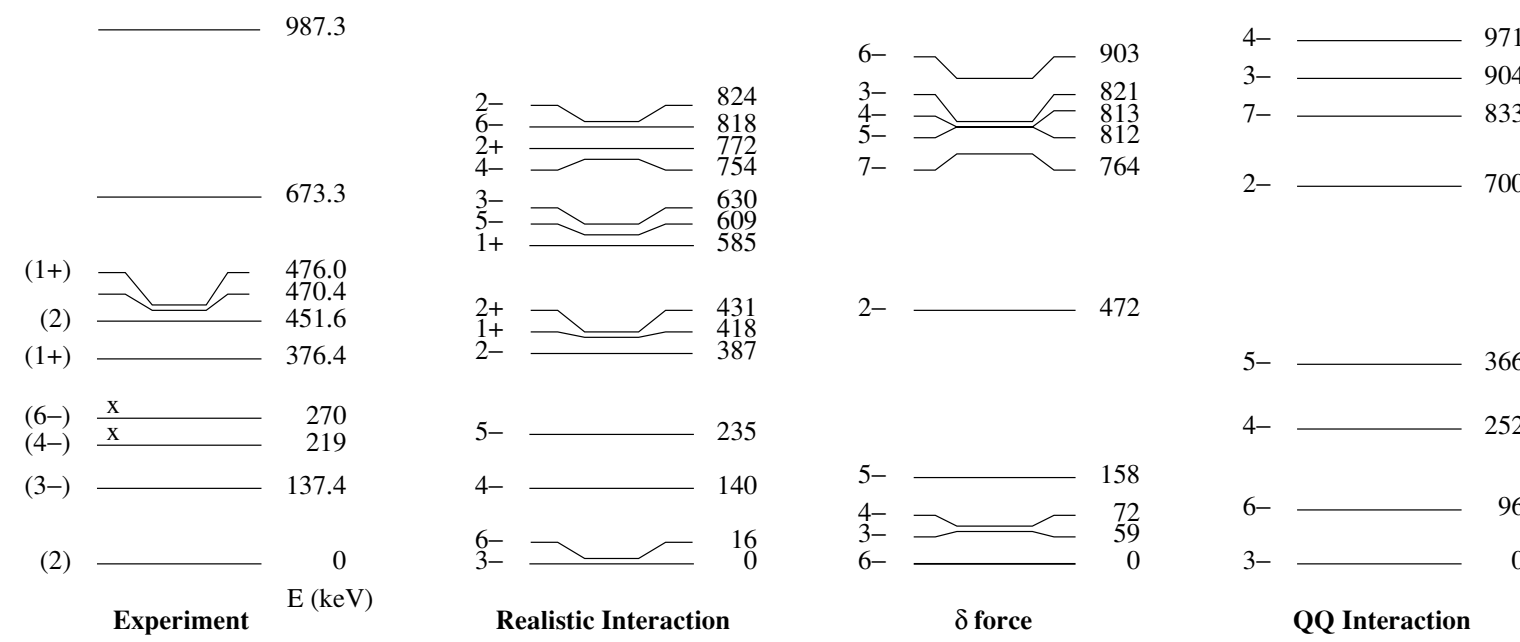

Realistic Interaction

FIG. 8. Nuclear structure of ${ }^{72} \mathrm{Cu}$ below $1 \mathrm{MeV}$ of excitation energy. Nuclear states observed experimentally are compared to theoretical spectra derived from (i) large-scale shell-model calculations within the $\left(2 p_{3 / 2} 1 f_{5 / 2} 2 p_{1 / 2} 1 g_{9 / 2}\right)$ shell space and using a realistic interaction and from (ii) a schematic approach using two types of residual proton-neutron interactions (a $\delta$ force and a quadrupole-quadrupole interaction). The states labeled with an "x" symbol are taken from [17]. 
The $\left(3^{-}\right),\left(4^{-}\right)$, and $\left(6^{-}\right)$states observed experimentally at low energy can be identified as members of the predicted (3-6) ${ }^{-}$multiplet. According to Table VII, they have a dominant (56\% to $64 \%$ ) $\pi 2 p_{3 / 2} \nu 1 g_{9 / 2}^{3}$ configuration. The energy difference between the states is quite well reproduced in the realistic approach. The three calculations performed in the present work predict a contraction of the (3-6)- multiplet when two neutrons are added to ${ }^{70} \mathrm{Cu}[8]$. It is difficult to address this question since the $5^{-}$member of the multiplet was not observed experimentally in ${ }^{72} \mathrm{Cu}$. From the work of Mach et al. [17], we can at least expect it to lie above the $\left(6^{-}\right)$state identified at $270 \mathrm{keV}$, which is in agreement with the present calculation.

The $\left(1^{+}\right)$state observed experimentally at $376 \mathrm{keV}$ can be identified as the first member of the $(1,2)^{+}$doublet of states. It is predicted by the realistic calculation to lie at $418 \mathrm{keV}$ with a dominant (more than $45 \%) \pi 2 p_{3 / 2} v\left(2 p_{1 / 2}^{-1} 1 g_{9 / 2}^{4}\right)$ configuration. The $I^{\pi}=2^{+}$member of the doublet is presumably one of the two $I=(2)$ states observed in the low-energy spectrum of ${ }^{72} \mathrm{Cu}$, namely the excited state at $452 \mathrm{keV}$ or the ground state of ${ }^{72} \mathrm{Cu}$. In the first case, the energy position of the $(1-2)^{+}$doublet would match pretty well with the large-scale shell-model prediction (see Fig. 8). However, if the $\left(2^{+}\right)$spin partner of the $\left(1^{+}\right)$state at $376 \mathrm{keV}$ is the ground state of ${ }^{72} \mathrm{Cu}$, the large splitting of the doublet could be explained by its strong mixing with another closely lying $2_{2}^{+}$state, having another dominant configuration such as $\pi 1 f_{5 / 2} v\left(2 p_{1 / 2}^{-1} 1 g_{9 / 2}^{4}\right)$. Such a $2_{2}^{+}$state was not observed experimentally, although it is predicted by the present calculation to lie at $772 \mathrm{keV}$. The calculation does not reproduce the desired effect, but the description of the odd-odd nuclei is usually not a simple task, since it is rather sensitive to the details of the two-body matrix elements.

Alternatively, one of the two $I=(2)$ states observed experimentally can be identified as the low-energy $2^{-}$member of the predicted $(2-7)^{-}$multiplet, having according to Table VII a dominant (more than 30\%) $\pi 1 f_{5 / 2} v 1 g_{9 / 2}^{3}$ configuration. In all theoretical approaches, the $2^{-}$state is expected to lie significantly below the other members of the multiplet: from about $130 \mathrm{keV}$ in the schematic calculation using a quadrupole-quadrupole interaction and up to almost $300 \mathrm{keV}$ in the realistic approach (see Fig. 8). In the latter case, the $2^{-}$state is expected at an excitation energy of $387 \mathrm{keV}$, which would be in good agreement with the $I=(2)$ state observed at $452 \mathrm{keV}$. However, the realistic interaction used in the present work predicts the $2^{-}$state in ${ }^{70} \mathrm{Cu}$ to lie at $755 \mathrm{keV}$, whereas it is observed at an excitation energy of $369 \mathrm{keV}$ [7], that is, $386 \mathrm{keV}$ below the expected value. If the same situation occurs in ${ }^{72} \mathrm{Cu}$, then the $I=(2)$ ground state of ${ }^{72} \mathrm{Cu}$ can be identified as the first member of the $(2-7)^{-}$ multiplet. Such a lowering of the $2^{-}$state could be justified by its leading configuration component, involving a $\pi 1 f_{5 / 2}$ valence proton coupled to three neutrons located in the $v 1 g_{9 / 2}^{3}$ orbital. The lowering of the $\pi 1 f_{5 / 2}$ proton orbital associated with the filling of the $v 1 g_{9 / 2}$ neutron orbital is indeed expected from earlier work on odd-copper isotopes [4,5]. In addition, a drop in energy of the $2^{-}$state with respect to the other members of a $(2-7)^{-}$multiplet of similar character is also observed in ${ }^{86} \mathrm{Rb}(Z=37, N=49)$, in which a $\pi 1 f_{5 / 2}$ proton is coupled to a $\nu 1 g_{9 / 2}$ neutron hole [33]. It is therefore unclear whether the predicted $2^{-}$state having a dominant $\pi 1 f_{5 / 2} \nu 1 g_{9 / 2}^{3}$ configuration is the one observed at $452 \mathrm{keV}$ or the ground state of ${ }^{72} \mathrm{Cu}$.

By now, the $3^{-}$to $7^{-}$members of the $(2-7)^{-}$multiplet have yet to be identified experimentally in ${ }^{72} \mathrm{Cu}$. They appear in the schematic approach below or slightly above $1 \mathrm{MeV}$, wheres they are found to be between $609 \mathrm{keV}\left(5_{2}^{-}\right)$and $941 \mathrm{keV}\left(7_{1}^{-}\right)$ in the realistic calculation. In the latter approach, they have a dominant $\pi 1 f_{5 / 2} \nu 1 g_{9 / 2}^{3}$ configuration, except the $5_{2}^{-}$state for which the $\pi 2 p_{3 / 2} \nu 1 \mathrm{~g}_{9 / 2}^{3}$ configuration prevails.

Except for the unexpected lowering of either the $\left(2_{1}^{+}\right)$or the $\left(2_{1}^{-}\right)$state for which theoretical explanations could be given, the theoretical calculations are in rather good agreement with the experimental results. The same overall features have been observed in ${ }^{70} \mathrm{Cu}$ [7]. In this lighter odd-odd $\mathrm{Cu}$ isotope, the ground state was identified as the $6^{-}$state belonging to the low-lying (3-6) $)^{-}$multiplet of $\pi 2 p_{3 / 2} \nu 1 g_{9 / 2}^{+1}$ configuration, and the $(1,2)^{+}$doublet associated with the $\pi 2 p_{3 / 2} v\left(2 p_{1 / 2}^{-1} 1 g_{9 / 2}^{2}\right)$ configuration was found at an excitation energy of about $300 \mathrm{keV}$. In ${ }^{72} \mathrm{Cu}$, the corresponding $\left(1^{+}\right)$state is located at a similar energy of $376 \mathrm{keV}$ and the (3-6) $)^{-}$multiplet is shifted upward because of the lowering of either the $\left(2_{1}^{+}\right)$or the $\left(2_{1}^{-}\right)$ state.

In [7], two high-energy $\left(1^{+}\right)$excited states have been identified at 1278 and $1980 \mathrm{keV}$ in ${ }^{70} \mathrm{Cu}$. The latter was interpreted as being dominantly due to the coupling of the valence proton and neutron in the $\pi 1 g_{9 / 2}$ and $\nu 1 g_{9 / 2}$ orbitals, respectively. In ${ }^{72} \mathrm{Cu}$, one of the two $\left(1^{+}\right)$states observed at about $2100 \mathrm{keV}$ may have a similar $\pi 1 g_{9 / 2} \nu 1 g_{9 / 2}^{3}$ configuration. As discussed in [7], the large-scale shell-model calculation using a realistic interaction, even the modified version discussed here, does not allow identification of any high-lying $1^{+}$excited state having a dominant $\pi 1 g_{9 / 2} \nu 1 g_{9 / 2}^{3}$ configuration. Nonetheless, such a state is well predicted within the schematic approach to lie at 2112 and $1998 \mathrm{keV}$ for a $\delta$ force and $Q Q$ interaction, respectively.

\section{B. Shell-model interpretation of the ${ }^{72} \mathrm{Ni} \rightarrow{ }^{72} \mathrm{Cu} \rightarrow{ }^{72} \mathrm{Zn}$ $\beta$-decay chain}

According to Fig. 6 , the $0^{+}$ground state of ${ }^{72} \mathrm{Ni}$ decays preferably toward the first $\left(1^{+}\right)$excited state of ${ }^{72} \mathrm{Cu}$ at $376 \mathrm{keV}[B . R .=42 \%, \log (f t)=4.8]$. Within the extreme single-particle shell-model picture, the decay mechanism can be interpreted as the transformation of a $\nu 2 p_{1 / 2}$ neutron into a $\pi 2 p_{3 / 2}$ proton.

Two main $\beta$-decay branches feed the $\left(1^{+}\right)$states of ${ }^{72} \mathrm{Cu}$ at 2060 and $2197 \mathrm{keV}(B . R .=11 \%$ and $7 \%, \log (f t)=4.6$ and 4.7 , respectively). As previously mentioned, one of these two states may be associated with a $\pi 1 g_{9 / 2} \nu 1 g_{9 / 2}^{3}$ configuration. It would therefore result from the conversion of a $v 1 \mathrm{~g}_{9 / 2}$ neutron into a $\pi \lg _{9 / 2}$ proton. The further de-excitation of this state to the $\left(2^{-}\right)$ground state of ${ }^{72} \mathrm{Cu}$ or to the $\left(2^{-}\right)$excited state at $452 \mathrm{keV}$ can then be interpreted as the lowering of the $\pi 1 g_{9 / 2}$ proton to the $\pi 1 f_{5 / 2}$ orbital across the $Z=40$ subshell gap.

The $\log (f t)$ values associated with the allowed GamowTeller transitions to the $1^{+}$states in ${ }^{72} \mathrm{Cu}$ were calculated 
TABLE VIII. Theoretical and experimental $\log (f t)$ values of the Gamow-Teller $\beta$ decay of ${ }^{72} \mathrm{Ni}$ to $\left(1^{+}\right)$excited states in ${ }^{72} \mathrm{Cu}$. The shell-model calculations have been performed with the realistic effective interaction described in the text.

\begin{tabular}{lrrrcc}
\hline \hline$I_{\exp }^{\pi}$ & $E_{\exp }(\mathrm{keV})$ & $I_{\text {th }}^{\pi}$ & $E_{\text {th }}(\mathrm{keV})$ & $\log (f t)_{\exp }$ & $\log (f t)_{\mathrm{th}}$ \\
\hline$\left(1^{+}\right)$ & $376.4(1)$ & $1_{1}^{+}$ & 418 & 4.7 & 4.6 \\
$\left(1^{+}\right)$ & $470.4(1)$ & $1_{2}^{+}$ & 585 & 5.1 & 5.1 \\
$\left(1^{+}\right)$ & $2060.4(1)$ & $1_{3}^{+}$ & 1534 & 4.6 & 4.6 \\
& & $1_{4}^{+}$ & 2273 & & 8.3 \\
$\left(1^{+}\right)$ & $2196.6(2)$ & $1_{5}^{+}$ & 2447 & 4.7 & 5.3 \\
\hline \hline
\end{tabular}

using the realistic interaction described here. They are reported in Table VIII and compared to the experimental results. The overall agreement is good, although an additional high-lying $1^{+}$state is predicted to be fed with a high $\log (f t)$ value of 8.3. However, even if it exists, such a state could not have been observed in the present experiment owing to its very low feeding in the $\beta$ decay of ${ }^{72} \mathrm{Ni}$.

The ground state of ${ }^{72} \mathrm{Cu}$ was interpreted here either as a $\left(2^{+}\right)$of a $\pi 2 p_{3 / 2} v\left(2 p_{1 / 2}^{-1} 1 g_{9 / 2}^{4}\right)$ configuration or as a $\left(2^{-}\right)$state with a dominant $\pi 1 f_{5 / 2} \nu 1 g_{9 / 2}^{3}$ component. In the first case, the $\beta$ decay of the ${ }^{72} \mathrm{Cu}$ ground state toward the $2_{1}^{+}$state in ${ }^{72} \mathrm{Zn}$ involves an allowed Gamow-Teller transition that can be interpreted as the conversion of a valence neutron occupying the $v 2 p_{1 / 2}$ orbital into an extra $\pi 2 p_{3 / 2}$ proton in ${ }^{72} \mathrm{Zn}$. The decay rates of the hypothetical $\left(2^{+}\right)$ground state of ${ }^{72} \mathrm{Cu}$ toward the first two $2^{+}$states of ${ }^{72} \mathrm{Zn}$ calculated in Fig. 7 are compared in Table IX to the experimental values. They are in rather good agreement with the measurements; the slight discrepancy is possibly related to the imposed restrictions on the model space.

However, if the ground state of ${ }^{72} \mathrm{Cu}$ has an $I=2^{-}$spin and parity, its decay toward the first two $2^{+}$and toward the first $\left(4^{+}\right)$excited states of ${ }^{72} \mathrm{Zn}$ would involve first-forbidden $\beta$ transitions of nonunique and unique character, respectively. According to the compilation of Singh et al. [24], the corresponding $\log (f t)$ values are expected to range between 5.1 and 11.0 for the two $\Delta I=0$ transitions and between 7.5 and 12.8 for the $\Delta I=2$ transition. Thus, the measured $\log (f t)$ values associated with the $\beta$ decay to the first two $2^{+}$states are compatible with an $I=2^{-}$ground state of ${ }^{72} \mathrm{Cu}$. As can be seen in Fig. 2 , the measured $\beta$-decay strength toward the $\left(4_{1}^{+}\right)$state is stronger that the compiled values, with a $\log (f t)$ value of 7.0. However, the associated absolute $\beta$ branching

TABLE IX. Theoretical and experimental $\log (f t)$ values of the $\beta$ transitions from ${ }^{72} \mathrm{Cu}$ ground state to the first $2^{+}$excited states in ${ }^{72} \mathrm{Zn}$. The shell-model calculations have been performed with the realistic effective interaction described in the text, assuming an $I=$ $2^{+}$ground state for ${ }^{72} \mathrm{Cu}$.

\begin{tabular}{lccccc}
\hline \hline$I_{\exp }^{\pi}$ & $E_{\exp }(\mathrm{keV})$ & $I_{\mathrm{th}}^{\pi}$ & $E_{\mathrm{th}}(\mathrm{keV})$ & $\log (f t)_{\exp }$ & $\log (f t)_{\mathrm{th}}$ \\
\hline $2^{+}$ & $652.68(5)$ & $2_{1}^{+}$ & 755 & 6.5 & 6.7 \\
$2^{+}$ & $1657.5(1)$ & $2_{2}^{+}$ & 1507 & 6.6 & 6.8 \\
\hline \hline
\end{tabular}

ratio is quite weak $\left[I_{\beta}=2.8(5) \%\right]$, and one cannot exclude an underestimation of the feeding to the $\left(4_{1}^{+}\right)$state by unobserved $\gamma$ transitions from upper lying excited states. As a conclusion, $\log (f t)$ arguments cannot be used to determine the parity of ${ }^{72} \mathrm{Cu}$ ground state.

\section{CONCLUSION}

The ${ }^{72} \mathrm{Ni} \rightarrow{ }^{72} \mathrm{Cu} \rightarrow{ }^{72} \mathrm{Zn} \beta$-decay chain has been studied in detail at the complementary facilities of LISOL (Centre de Recherche du Cyclotron, Belgium) and ISOLDE (CERN, Switzerland). Conventional $\beta-\gamma$ detection techniques associated with a selective laser ionization of $\mathrm{Ni}$ and $\mathrm{Cu}$ atoms led to detailed level schemes for ${ }^{72} \mathrm{Cu}$, for which a half-life $T_{1 / 2}=6.63(3) \mathrm{s}$ was measured, and ${ }^{72} \mathrm{Zn}$. In contrast to ${ }^{70} \mathrm{Cu}$, an in-source laser spectroscopy study revealed no $\beta$-decaying isomeric states in ${ }^{72} \mathrm{Cu}$.

The nuclear structure of ${ }^{72} \mathrm{Cu}$ at low excitation energy was discussed in terms of single-particle coupling between the valence protons and neutrons. Two shell-model calculations were performed using different modelization of the effective residual proton-neutron interaction. They revealed that the low-lying states in ${ }^{72} \mathrm{Cu}$ result predominantly from the coupling of the valence proton occupying the $\pi 2 p_{3 / 2}$ or $\pi 1 f_{5 / 2}$ orbitals to the valence neutrons located either in the $v 1 g_{9 / 2}$ or in the $v 2 p_{1 / 2}$ orbital. Most of the low-energy states observed in ${ }^{72} \mathrm{Cu}$ could be attributed to the $(3-6)^{-},(1-2)^{+}$, and $(2-7)^{-}$ multiplets of states already identified in ${ }^{70} \mathrm{Cu}$.

In contrast to ${ }^{70} \mathrm{Cu}$, for which the ground state spin is $I^{\pi}=(6)^{-}$, the $\beta$-decay studies of ${ }^{72} \mathrm{Ni}$ and ${ }^{72} \mathrm{Cu}$ allowed assignment of a spin value $I=(2)$ to the ground state of ${ }^{72} \mathrm{Cu}$. Two theoretical explanations were proposed to justify such a change. In both cases, an unexpected drop in energy of either a $\left(2^{+}\right)$state of $\pi 2 p_{3 / 2} \nu\left(2 p_{1 / 2}^{-1} 1 g_{9 / 2}^{4}\right)$ configuration or a $\left(2^{-}\right)$state of $\pi 1 f_{5 / 2} \nu 1 g_{9 / 2}^{3}$ dominant component was invoked. The first scenario involves a strong mixing of the $\left(2_{1}^{+}\right)$ state with another $\left(2^{+}\right)$state having a $\pi 1 f_{5 / 2} \nu\left(2 p_{1 / 2}^{-1} 1 g_{9 / 2}^{4}\right)$ configuration. The second one suggests a dramatic lowering of the $\pi 1 f_{5 / 2}$ proton orbital as compared to ${ }^{70} \mathrm{Cu}$, owing to the addition of two neutrons in the $v 1 g_{9 / 2}$ orbital. These observations highlight the relevance of the nuclear structure study of neutron-rich copper isotopes and the need for a better understanding of the residual protonneutron interaction in the $N=40-50$ region. Specifically, the ground-state spin and parity of ${ }^{72} \mathrm{Cu}$ could be investigated by means of laser spectroscopy or Coulomb excitation studies.

\section{ACKNOWLEDGMENTS}

This work was performed within the frame of the IS365 Collaboration and the ISOLDE Collaboration. We gratefully thank J. Gentens and P. Van den Bergh for running the LISOL separator and the ISOLDE technical group for assistance during the experiment performed at CERN. N.A.S. thanks E. Caurier and F. Nowacki (IPHC, Strasbourg) for making available the ANTOINE code and the interaction used here. 
J.-C.T thanks M.-G. Porquet (CSNSM, Orsay) and O. Sorlin (GANIL, Caen) for fruitful discussions. This work was supported by the Inter-University Attraction Poles (IUAP) Re- search Program No. P5/07 and the European Union (Contract No. ERBFMGEECT980120). K.V.d.V. is a Research Assistant of the FWO-Vlaanderen.
[1] A. M. Oros-Peusquens and P. F. Mantica, Nucl. Phys. A669, 81 (2000).

[2] B. Pfeiffer et al., Nucl. Phys. A693, 282 (2001).

[3] K. Langanke et al., Phys. Rev. Lett. 90, 241102 (2003).

[4] N. A. Smirnova, A. De Maesschalck, A.Van Dyck, and K. Heyde, Phys. Rev. C 69, 044306 (2004).

[5] S. Franchoo et al., Phys. Rev. C 64, 054308 (2001).

[6] J. Van Roosbroeck et al., Phys. Rev. Lett. 92, 112501 (2004).

[7] J. Van Roosbroeck et al., Phys. Rev. C 69, 034313 (2004).

[8] J. Van Roosbroeck et al., Phys. Rev. C 71, 054307 (2005).

[9] J.-C. Thomas et al. (in preparation).

[10] E. Runte et al., Nucl. Phys. A399, 163 (1983).

[11] F. R. Hudson and R. N. Glover, Nucl. Phys. A189, 264 (1972).

[12] A. N. Wilson et al., Eur. Phys. J. A 9, 183 (2000).

[13] P. Armbruster et al., Europhys. Lett. 4, 793 (1987).

[14] S. Franchoo et al., Phys. Rev. Lett. 81, 3100 (1998).

[15] M. Bernas et al., Z. Phys. A 336, 41 (1990).

[16] R. Grzywacz et al., Phys. Rev. Lett. 81, 766 (1998).

[17] H. Mach, in Proceedings of the International Symposium on Nuclear Structure Physics, Göttingen, Germany, 2001 (World Scientific, Singapore, 2001), p. 379.

[18] M. Stanoiu, Ph.D. thesis, Université de Caen/Basse-Normandie, France, 2003, http://tel.ccsd.cnrs.fr/documents/archives0/00/00/ $27 / 75 /$
[19] S. Franchoo, Ph.D. thesis, Katholieke Universiteit Leuven, Leuven, Belgium, 1999.

[20] J. Van Roosbroeck, Ph.D. thesis, Katholieke Universiteit Leuven, Leuven, Belgium, 2002, http://fys.kuleuven.be/iks/ lisol/publications/thesis.htm

[21] G. Audi et al., Nucl. Phys. A729, 3 (2003).

[22] D. Karamanis, Nucl. Instrum. Methods A 505, 282 (2003).

[23] GEANT, CERN Program Library Long Writeup W5013, CERN, Geneva, 1993.

[24] B. Singh et al., Nucl. Data Sheets 84, 487 (1998).

[25] W.-T. Chou and M. M. King, Nucl. Data Sheets 73, 215 (1994).

[26] http://ie.lbl.gov/toi2003/MassSearch.asp

[27] M. Facina (private communication).

[28] R. Firestone, Table of Isotopes, 8th ed. (Wiley, New York, 1996).

[29] E. Caurier and F. Nowacki, Acta Phys. Pol. B 30, 705 (1999).

[30] M. Hjorth-Jensen, T. T. S. Kuo, and E. Osnes, Phys. Rep. 261, 125 (1995).

[31] F. Nowacki, Ph.D. thesis, Université Louis Pasteur, Strasbourg, France, 1996.

[32] N. A. Smirnova et al. (in preparation).

[33] P. Li et al., Nucl. Phys. A469, 393 (1987). 